OPEN ACCESS

Edited by:

Pedro Antonio Valdes-Sosa Joint China Cuba Lab for Frontiers

Reaearch in Translational

Neurotechnology, Cuba

Reviewed by:

Srikantan S. Nagarajan, University of California, San Francisco,

Gabriele Arnulfo, University of Genoa, Italy Andreas Horn,

Harvard Medical School, USA

*Correspondence: Alejandro O. Blenkmann ablenkmann@gmail.com

${ }^{\dagger}$ Joint last author.

Received: 29 November 2016 Accepted: 01 February 2017 Published: 02 March 2017

Citation:

Blenkmann AO, Phillips HN Princich JP, Rowe JB, Bekinschtein TA, Muravchik $\mathrm{CH}$ and Kochen S (2017) iElectrodes: A Comprehensive Open-Source Toolbox for Depth and Subdural Grid Electrode Localization.

Front Neuroinform 11:14 doi: 10.3389/fninf.2017.00014

\section{iElectrodes: A Comprehensive Open-Source Toolbox for Depth and Subdural Grid Electrode Localization}

\author{
Alejandro O. Blenkmann 1, 2,3,4*, Holly N. Phillips ${ }^{5,6}$, Juan P. Princich ${ }^{2}$, James B. Rowe ${ }^{5,6}$, \\ Tristan A. Bekinschtein ${ }^{7}$, Carlos H. Muravchik ${ }^{8 \dagger}$ and Silvia Kochen ${ }^{2,3,4 \dagger}$ \\ 'FRONT Neurolab, Department of Psychology, University of Oslo, Oslo, Norway, ${ }^{2}$ Estudios de Neurociencias y Sistemas \\ Complejos, CONICET- El Cruce Hospital - Universidad Nacional Arturo Jauretche, Buenos Aires, Argentina, ${ }^{3}$ Institute of \\ Cellular Biology and Neuroscience "Prof E. De Robertis," School of Medicine, University of Buenos Aires - CONICET, Buenos \\ Aires, Argentina, ${ }^{4}$ Epilepsy Section, Division of Neurology, Ramos Mejia Hospital, Buenos Aires, Argentina, ${ }^{5}$ Department of \\ Clinical Neurosciences, University of Cambridge, Cambridge, UK, ${ }^{6}$ MRC Cognition and Brain Sciences Unit, Cambridge, UK, \\ ${ }^{7}$ Department of Psychology, University of Cambridge, Cambridge, UK, ${ }^{8}$ Facultad de Ingeniería, Instituto de Investigaciones \\ en Electrónica, Control y Procesamiento de Señales, Universidad Nacional de La Plata, La Plata, Argentina
}

The localization of intracranial electrodes is a fundamental step in the analysis of invasive electroencephalography (EEG) recordings in research and clinical practice. The conclusions reached from the analysis of these recordings rely on the accuracy of electrode localization in relationship to brain anatomy. However, currently available techniques for localizing electrodes from magnetic resonance (MR) and/or computerized tomography (CT) images are time consuming and/or limited to particular electrode types or shapes. Here we present iElectrodes, an open-source toolbox that provides robust and accurate semi-automatic localization of both subdural grids and depth electrodes. Using pre- and post-implantation images, the method takes 2-3 min to localize the coordinates in each electrode array and automatically number the electrodes. The proposed pre-processing pipeline allows one to work in a normalized space and to automatically obtain anatomical labels of the localized electrodes without neuroimaging experts. We validated the method with data from 22 patients implanted with a total of 1,242 electrodes. We show that localization distances were within $0.56 \mathrm{~mm}$ of those achieved by experienced manual evaluators. iElectrodes provided additional advantages in terms of robustness (even with severe perioperative cerebral distortions), speed (less than half the operator time compared to expert manual localization), simplicity, utility across multiple electrode types (surface and depth electrodes) and all brain regions.

Keywords: SEEG, ECoG, intracranial EEG, MRI, CT, atlas, epilepsy

\section{INTRODUCTION}

Human intracranial EEG (iEEG) recordings provide a unique contribution to cognitive neuroscience, allowing brain activity to be measured with high spatial and temporal resolution (Mukamel and Fried, 2012). Typically, subdural grids and depth electrodes are implanted in epilepsy patients undergoing pre-surgical evaluation, to characterize the areas involved in the genesis and propagation of seizures, i.e., the epileptogenic zone and eloquent cortex (Chauvel et al., 1987; Rosenow and Lüders, 2001; Kochen et al., 2002). 
The spatial precision of iEEG recordings relies on accurate localization in relationship to brain anatomy (Lachaux et al., 2003). In clinical practice, accurate and robust localization of electrodes is essential for identifying the epileptogenic zone for resection (Pieters et al., 2013; Princich et al., 2013; Taimouri et al., 2014) and relating these findings to previous cognitive function tests, structural lesions, and other neuroimaging studies which are typically expressed in a standard anatomic space (McGonigal et al., 2007; Princich et al., 2013). In research, localizing electrodes within a standardized brain space also allows comparisons across subjects for group-level analysis (Keller et al., 2011; Kadipasaoglu et al., 2014) and enables direct comparison of single patient recordings to non-invasive imaging studies (Chennu et al., 2013; Phillips et al., 2016).

Many methods have been developed to locate implanted electrodes. Though some methods solely use MRI images to identify electrode locations (Kovalev et al., 2005; Ball et al., 2009; Gaillard et al., 2009; Axmacher et al., 2010; Yang et al., 2012), most methods also incorporate post-implantation CT images to obtain greater localization accuracy (Bootsveld et al., 1994; Van Rooijen et al., 2013). Currently available methods suffer from significant limitations, which we aimed to address with a new semi-automatic method.

Previous studies that co-register pre- or post-implantation MRI with CT images, utilize the tissue contrasts in the MRI to identify the anatomical positions of the electrodes which are seen more clearly in the CT images (Lachaux et al., 2003). Commonly, electrode positions are manually identified through observation of the associated $\mathrm{CT}$ artifacts in $2 \mathrm{D}$ views or $3 \mathrm{D}$ rendered brain images (Winkler et al., 2000; Tao et al., 2009; Princich et al., 2013). Manual identification is widely used, especially in clinical practice, but it is time consuming to identify and record the anatomical location of every electrode and requires detailed knowledge of MRI based neuroanatomy (Princich et al., 2013). To overcome the former drawback, we previously proposed the use of a brain atlas to make anatomical labeling fast and simple (Princich et al., 2013).

Studies co-registering post-implantation CT images to preimplantation MRI images usually lead to miss-localization of grid electrodes by up to $14 \mathrm{~mm}$ from the brain surface because of fluid build-up around implanted grids (Dalal et al., 2008). To address this problem, localized electrodes can be projected in an orthogonal direction to the brain surface (Hermes et al., 2010; Taimouri et al., 2014) or fitted to the closest brain surface using a constrained energy-minimization algorithm in a spring like grid (Dykstra et al., 2012). Alternatively, methods that localize grid electrodes on post-implantation MRI and CT images do not need corrections since both images contain the same tissuedeformation (Winkler et al., 2000; LaViolette et al., 2011b; Ibáñez et al., 2013; Azarion et al., 2014).

In studies using only depth electrodes there is typically less tissue deformation. In 66 patients undergoing deep brain stimulation, Elias et al. (2007) observed a mean displacement of less than $1 \mathrm{~mm}$ in anterior commissure and posterior commissure coordinates and $3.5 \mathrm{~mm}$ displacement in the frontal poles due to stereotactic procedures. Thus, it can be beneficial in these situations to use pre-implantation MRI images without electrode artifacts and coregister them to post-implantation CT images (Ekstrom et al., 2008; Princich et al., 2013; Arnulfo et al., 2015).

Other methods use intra-operative photographs, which are registered with a pre-implantation MRI to localize subdural grids visible in the craniotomy (Wellmer et al., 2002; Pieters et al., 2013). Dalal et al. (2008) took the further step of also using X-ray registered images to localize grids not visible in the photographs, however this procedure requires several hours to be completed.

The localization methods described above require the manual selection of individual electrodes in the CT, MRI, or photographs (Dalal et al., 2008; Hermes et al., 2010; Dykstra et al., 2012; Yang et al., 2012; Pieters et al., 2013). Manual procedures are time consuming and prone to operator errors, thus semi-automatic and automatic localization methods have been developed, for example based on spatial filters (Sebastiano et al., 2006; Taimouri et al., 2014) or previous planning information (Arnulfo et al., 2015).

Most techniques are constrained to either localizing subdural grids or depth electrodes (Sebastiano et al., 2006; Dalal et al., 2008; Hermes et al., 2010; Yang et al., 2012; Pieters et al., 2013). However, it is not uncommon that specialized epilepsy centers use both subdural and depth electrodes in combination, in single patients, or in separate patients (Gonzalez-Martinez et al., 2013; Vadera et al., 2013; Enatsu et al., 2014). Moreover, the combination has increased in the last decade (Moshé et al., 2015) calling for a unified method of localization.

Here we present iElectrodes, a toolbox to localize both subdural grids and depth electrodes, and a pre-processing pipeline for MRI and CT images. The toolbox and pipeline were designed to meet the following objectives: usable in standardized anatomical space; speed; minimization of operator expertise dependency; and to be reliable across patients, locations, and electrode types. Additionally, we implemented an atlas-based anatomical description of each electrode location to facilitate interpretation and reporting and we developed an automated procedure to designate the electrode order. Finally, we made this toolbox an open-source application for the research and clinical community.

\section{MATERIALS AND METHODS}

\section{Patients}

Depth electrodes, grids or strips are commonly used in the clinical diagnosis protocol for the surgical treatment of drugresistant epilepsy patients (Kochen et al., 2002; Kwan et al., 2010). We prospectively recruited 22 consecutive patients (7 female, mean age 29 years) with drug-resistant epilepsy, who were candidates for respective surgery. Intracranial electrodes were temporarily implanted for 5-10 days while iEEG and video were continuously recorded. MRI and CT images were acquired as part of the clinical procedure. The pre-surgical evaluation aimed to localize the epileptogenic zone and to delineate eloquent cortex (Rosenow and Lüders, 2001). Three patients were implanted with subdural grids, nine with subdural grids and depth electrodes, and eleven were exclusively implanted with depth electrode arrays (patients numbered from 1 to 3,4 to 11 , and 12 to 22 respectively). The study was conducted with the approval of 
the Research Ethics Committee of Ramos Mejía Hospital and El Cruce Hospital in accordance with the ethical standards laid down in the 1964 Declaration of Helsinki. All patients gave their written informed consent for the participation in this study and the use of their collected information.

\section{Electrode Arrays}

Cortical surface grids array dimensions were $2 \times 4,2 \times 8$, $4 \times 5,4 \times 8,6 \times 8$, and $8 \times 8$ contacts. Electrode strip dimensions were $1 \times 4$ contacts. Electrode contacts were made of platinum embedded in a $0.5 \mathrm{~mm}$ flexible silicon plate, with $4 \mathrm{~mm}$ diameter, $2.3 \mathrm{~mm}$ exposure diameter and $10 \mathrm{~mm}$ intercontact center to center spacing (Ad-Tech Medical Instrument Corporation, USA). In some cases, a platinum marker was present in between contacts 1 and 2 .

Depth electrode arrays had: (a) 8 or 10 platinum contacts with 5 or $10 \mathrm{~mm}$ inter-contact center to center distance, contact length of $2.4 \mathrm{~mm}$ and $1.1 \mathrm{~mm}$ diameter, or (b) 9 platinum contacts, $3 \mathrm{~mm}$ distance between the first and the second contact and $6 \mathrm{~mm}$ inter-electrode distance from the second to the last. Contact length was $1.57 \mathrm{~mm}$ and the electrode diameter was $1.28 \mathrm{~mm}$. Simultaneously, 9 micro-wire platinum electrodes were implanted through the lumen of the electrode array. Each microwire had a diameter of $38 \mu \mathrm{m}$ and was trimmed at $\sim 4 \mathrm{~mm}$ past the tip (Ad-Tech Medical Instrument Corporation, USA).

The implantation sites were solely based on clinical criteria and had no relationship to the current study. Table 1 shows electrode implantation descriptions and anatomical locations for each patient. Due to the different targets achieved with deep electrode arrays, in some cases not all contacts were placed inside the brain and some remained in the skull or cerebrospinal fluid. Since those contacts were not electrophysiologically relevant, they were excluded from the current study.

\section{Acquisition of MRI and CT Images}

T1-weighted MRI FFE sequence with $1 \mathrm{~mm}$ isotropic resolution images were acquired within 2 days after implantation in patients with subdural grids (with or without depth electrodes, patients 1-11, Achieva, Phillips Medical Systems, The Netherlands, 1.5 $\mathrm{T}$ magnet unit, $\mathrm{TR} / \mathrm{TE} / \mathrm{TI}=9.2 / 4.2 / 450 \mathrm{~ms}$, matrix $256 \times 256$, FOV $256 \times 256 \mathrm{~mm}$, slice thickness $1 \mathrm{~mm}$, and 175 slices), and prior to implantation in patients with depth electrodes only (patients 12-22, Achieva, Phillips Medical Systems, The Netherlands, $3 \mathrm{~T}$ magnet unit, TR/TE $=6.9 / 3.2 \mathrm{~ms}$, matrix $256 \times 256$, FOV $256 \times 256 \mathrm{~mm}$, slice thickness $1 \mathrm{~mm}$, and 180 slices). Special care was taken when acquiring MRI images with intracranial electrodes. Each multi-channel connection lead was keep straight and without touching any other lead, forming no loops which could potentially induce currents (Davis et al., 1999). MRI of implanted patients has been shown to be safe, with respect to possible movements induced by electromagnetic fields and heating of electrodes (Davis et al., 1999; Carmichael et al., 2008; Princich et al., 2013; Azarion et al., 2014).

CT scans for each patient were performed within 2 days after electrode implantation to visualize electrode locations and as part of the clinical protocol for the evaluation of possible complications such as hematoma, contusions or subdural effusions. CT images (Emotion, Siemens Medical Solutions, Germany, $120 \mathrm{kVp}, 240 \mathrm{~mm}$ FOV, $512 \times 512$ matrix, $0.6 \mathrm{~mm}$ slice thickness for patients 1-11, or Aquilion, Toshiba Medical Systems, Japan, 120 kVp, 220-230 mm FOV, $512 \times 512$ matrix and $0.5 \mathrm{~mm}$ slice thickness for patients 12-22) were reconstructed to isotropic $1 \mathrm{~mm}$ resolution.

\section{Image Pre-processing}

Figure 1 shows an overview of the pre-processing pipeline. The acquired MRI and CT images were exported in DICOM format and then transformed to the NIfTI standard using $\mathrm{dcm} 2$ nii software (MRIcron, USA). Pre-processing requires the coregistration of the images before they can be processed using the iElectrodes toolbox. We also normalized the images to a standard space as part of the pre-processing pipeline. However, this step can be omitted if a native space representation is required, as it is in some clinical practice routines (Princich et al., 2013).

Although we have implemented the pre-processing steps using a particular set of image analysis softwares, pre-processing can be performed using other available packages. The ability to adopt alternative normalization procedures will be of particular interest, in view of the marked peri-operative cerebral deformations arising with subdural grids.

\section{MRI Cortical Segmentation}

We performed subject-specific cortical segmentation using the Freesurfer v5.0 image analysis suite (Dale and Sereno, 1993; Dale et al., 1999), obtaining a segmented image of the cerebral cortex, based on gyral and sulcal structures (Figure 1B; Fischl et al., 2004; Desikan et al., 2006).

\section{MRI and CT Coregistration}

CT images were coregistered to MRI images (Figure 1C) in SPM8 toolbox (Wellcome Trust Centre for Neuroimaging, UCL) using a 6-parameter rigid-body transformation based on the maximization of the normalized mutual information (Studholme et al., 1998), which has shown to perform well for the coregistration of MRI and CT image modalities (Ken et al., 2007; Hermes et al., 2010; Azarion et al., 2014). Coregistered images were visually checked.

\section{Spatial Normalization}

MRI images were normalized to the Montreal Neurological Institute (MNI152) standard space in SPM8 (Figure 1D). Brain masks obtained from Freesurfer were used in the normalization procedure to avoid unwanted deformations due to non-standard tissue (see Supplementary Figures 1A,B). Normalization was performed in two steps: (a) bias correction and (b) nonlinear coregistration (Ashburner and Friston, 1999). This process includes non-linear warping transformations to account for the large deformations observed due to the surgical procedure (see Figure 1A). The same non-linear transformation was applied to the coregistered CT images (Figure 1D). Other 
TABLE 1 | Implantation details.

\begin{tabular}{|c|c|c|c|c|c|c|c|c|c|c|c|c|c|c|}
\hline \multirow{2}{*}{$\begin{array}{l}\text { Patient } \\
\text { no. }\end{array}$} & \multirow{2}{*}{ Gender } & \multirow[t]{2}{*}{ Age } & \multicolumn{3}{|c|}{ Subdural grids } & \multicolumn{3}{|c|}{ Depth electrodes } & \multicolumn{6}{|c|}{ Brain areas } \\
\hline & & & Hem & $\mathbf{N}$ & Arrays & Hem & $\mathbf{N}$ & Arrays & $\mathbf{F}$ & $\mathbf{T}$ & $\mathbf{P}$ & 0 & I & $\mathbf{L}$ \\
\hline 1 & $\mathrm{~F}$ & 20 & $\mathrm{R}$ & 64 & $(8 \times 8)$ & & & & & $\checkmark$ & $\checkmark$ & $\checkmark$ & & \\
\hline 2 & M & 33 & $L$ & 64 & $(8 \times 8)$ & & & & $\checkmark$ & $\checkmark$ & $\checkmark$ & & & \\
\hline 3 & $\mathrm{M}$ & 23 & $\mathrm{R}$ & 48 & $\begin{array}{c}(4 \times 8) ;(2 \times 4) \\
4 ; 4\end{array}$ & & & & $\checkmark$ & & & & & \\
\hline 4 & $\mathrm{M}$ & 59 & $L$ & 64 & $(8 \times 8)$ & $\mathrm{L} / \mathrm{R}$ & 20 & $10 / 10$ & $\checkmark$ & $\checkmark$ & $\checkmark$ & $\checkmark$ & & $\checkmark$ \\
\hline 5 & $\mathrm{M}$ & 29 & $L$ & 64 & $(4 \times 8) ;(4 \times 8)$ & $L$ & 23 & $9 ; 9 ; 5$ & $\checkmark$ & $\checkmark$ & $\checkmark$ & & & \\
\hline 6 & $\mathrm{M}$ & 44 & $\mathrm{R}$ & 24 & $(4 \times 5) ; 4$ & L/R & 20 & $10 / 10$ & $\checkmark$ & $\checkmark$ & & $\checkmark$ & & $\checkmark$ \\
\hline 7 & $\mathrm{M}$ & 29 & $\mathrm{R}$ & 28 & $(4 \times 5) ; 4 ; 4$ & $\mathrm{R}$ & 20 & $10 ; 10$ & $\checkmark$ & $\checkmark$ & & $\checkmark$ & & $\checkmark$ \\
\hline 8 & $\mathrm{~F}$ & 30 & $L$ & 48 & $(6 \times 8)$ & $L$ & 29 & $10 ; 5 ; 5 ; 9$ & $\checkmark$ & $\checkmark$ & $\checkmark$ & $\checkmark$ & & $\checkmark$ \\
\hline 9 & $\mathrm{M}$ & 20 & $\mathrm{R}$ & 104 & $\begin{array}{c}(8 \times 8) ;(2 \times 8) \\
(2 \times 8) ; 4 ; 4\end{array}$ & $\mathrm{R}$ & 8 & $4 ; 4$ & $\checkmark$ & $\checkmark$ & $\checkmark$ & $\checkmark$ & & \\
\hline 10 & $\mathrm{~F}$ & 44 & $L$ & 52 & $\begin{array}{c}(4 \times 8) ; 4 ; 4 ; 4 \\
4 ; 4\end{array}$ & $L$ & 10 & $5 ; 5$ & $\checkmark$ & $\checkmark$ & & & & $\checkmark$ \\
\hline 11 & $\mathrm{~F}$ & 24 & $\mathrm{~L}$ & 52 & $(6 \times 8) ; 4$ & $\mathrm{~L}$ & 12 & $6 ; 6$ & $\checkmark$ & & $\checkmark$ & & & \\
\hline 12 & M & 26 & & & & $L$ & 31 & $9 ; 9 ; 7 ; 6$ & $\checkmark$ & $\checkmark$ & $\checkmark$ & & $\checkmark$ & \\
\hline 13 & $\mathrm{M}$ & 20 & & & & L/R & 23 & $3 ; 7 ; 6 ; 2 / 5$ & & $\checkmark$ & & $\checkmark$ & & $\checkmark$ \\
\hline 14 & $\mathrm{~F}$ & 22 & & & & $\mathrm{R}$ & 44 & $9 ; 9 ; 8 ; 9 ; 9$ & $\checkmark$ & $\checkmark$ & & & $\checkmark$ & $\checkmark$ \\
\hline 15 & M & 19 & & & & L/R & 47 & 7; 8; 8/7; 8; 9 & & $\checkmark$ & & & & $\checkmark$ \\
\hline 16 & $\mathrm{~F}$ & 49 & & & & $\mathrm{~L} / \mathrm{R}$ & 56 & $\begin{array}{c}\text { 5/7; 6; 9; 6; 3; } \\
7 ; 7 ; 6\end{array}$ & & $\checkmark$ & $\checkmark$ & $\checkmark$ & & $\checkmark$ \\
\hline 17 & $\mathrm{M}$ & 19 & & & & $L$ & 36 & $7 ; 7 ; 7 ; 7 ; 8$ & $\checkmark$ & $\checkmark$ & & & & $\checkmark$ \\
\hline 18 & $\mathrm{M}$ & 21 & & & & L/R & 47 & 8; 8; 8/8; 7; 8 & & $\checkmark$ & & & & $\checkmark$ \\
\hline 19 & $\mathrm{M}$ & 25 & & & & $\mathrm{R}$ & 46 & $7 ; 7 ; 7 ; 9 ; 7 ; 9$ & & $\checkmark$ & & $\checkmark$ & & $\checkmark$ \\
\hline 20 & $\mathrm{M}$ & 37 & & & & $\mathrm{~L} / \mathrm{R}$ & 48 & 8; 8; 7/8; 8; 8 & & $\checkmark$ & & & & $\checkmark$ \\
\hline 21 & M & 33 & & & & $L$ & 42 & $7 ; 10 ; 7 ; 4 ; 7 ; 7$ & $\checkmark$ & $\checkmark$ & $\checkmark$ & & & $\checkmark$ \\
\hline 22 & $F$ & 23 & & & & $\mathrm{R}$ & 68 & $\begin{array}{c}9 ; 10 ; 7 ; 10 ; 8 \\
10 ; 7 ; 7\end{array}$ & $\checkmark$ & $\checkmark$ & $\checkmark$ & & & $\checkmark$ \\
\hline
\end{tabular}

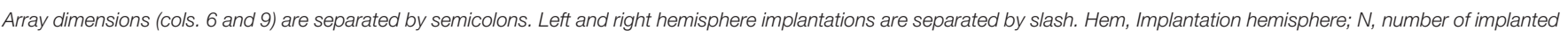

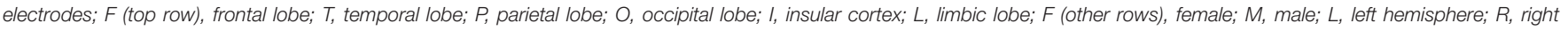
hemisphere.

normalization procedures may be adopted for use with iElectrodes.

\section{Brain Segmentation of Normalized Brain}

Brain segmentation masks were obtained from normalized MRI images using FMRIB software library (FSL, Oxford, UK) Brain Extraction Tool (BET) (Smith, 2002; Jenkinson et al., 2005; Figure 1E). These brain masks were useful in circumscribing the regions where intracranial electrodes were localized.

\section{Localization of Intracranial Electrodes in iElectrodes Toolbox}

Coregistered and normalized MRI and CT images, as well as brain masks, were loaded into the iElectrodes toolbox in NIfTI format. The toolbox was implemented in MATLAB (The Mathworks Inc., USA) using a graphical user interface, and includes in-house designed algorithms and functions from other publicly available MATLAB toolboxes (see details in the acknowledgments). The sequence of steps to localize, number, and label all electrodes is described below. In brief, all voxels corresponding to intracranial electrodes were obtained by masking and thresholding the CT. Then, electrodes coordinates were determined as the center of mass of each cluster of voxels. Finally, electrodes were numbered and anatomical labels assigned to each one using a probabilistic atlas. From the user perspective, the localization procedure is fast and straightforward. Users are required to manually determine the brain mask size and the threshold value, and select all voxels corresponding to a single array. Finally the user must run the automatic localization, numbering, and anatomical labeling algorithms. Two sample videos demonstrate the localization procedure: Supplementary Video 1 (subdural grid) and Supplementary Video 2 (depth electrodes). 


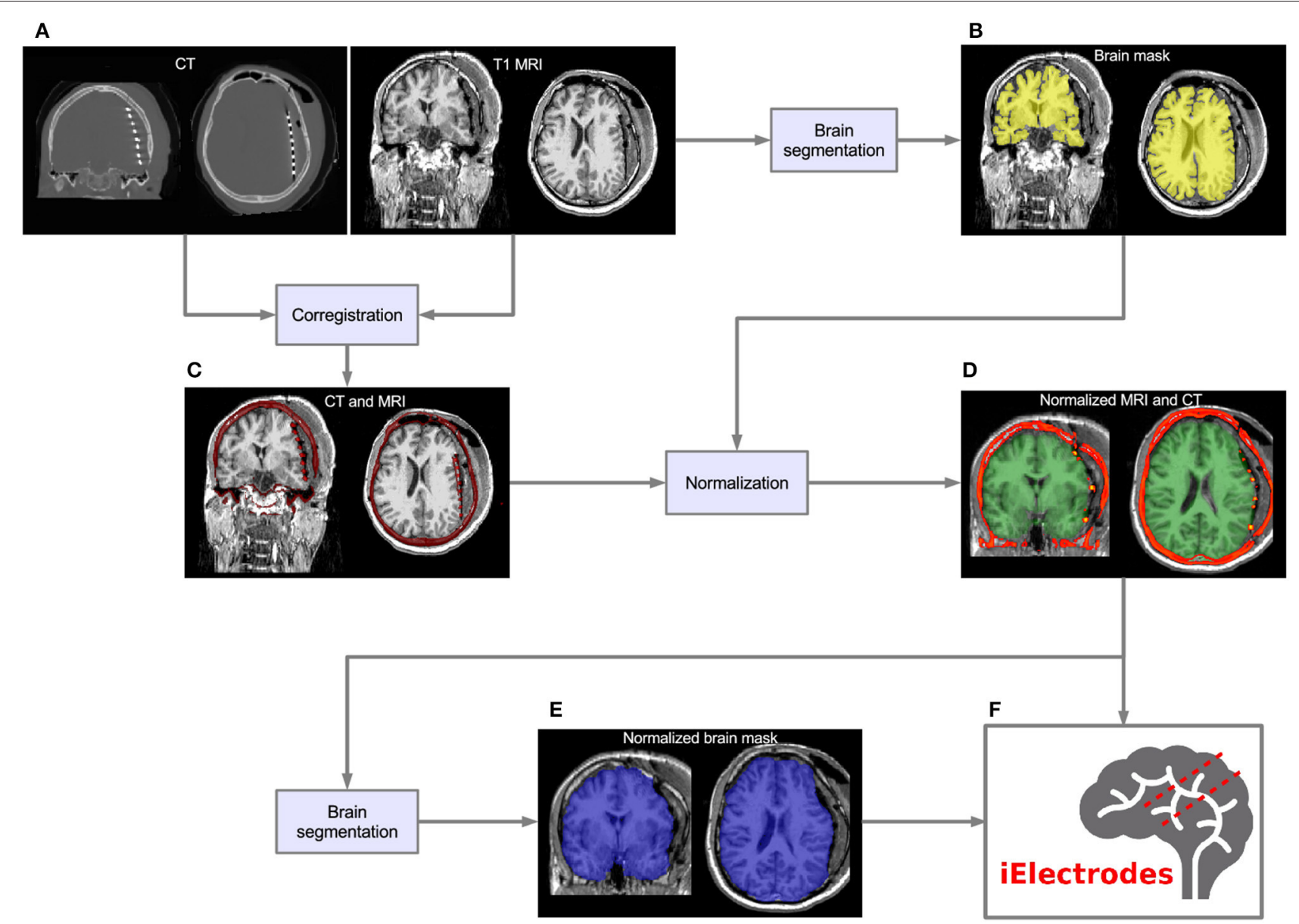

FIGURE 1 | Pre-processing pipeline. (A) Acquired images, CT and T1 MRI, showing electrode artifacts, brain shift and compression caused by edema. (B) A brain mask (yellow) is obtained by segmenting the MRI. Notice that the mask border follows the brain surface accurately. (C) MRI and CT images are coregistered in native space. Observe the electrode artifacts in the thresholded CT (red) over the MRI. (D) Using the previous brain mask (yellow), the MRI is spatially normalized and the same transformation applied to the CT. Observe the CT (red) on top of the normalized brain MRI. For illustrative purposes we also show the MNI average brain mask (green). (E) A normalized brain mask is obtained (blue) from the normalized MRI. (F) Normalized MRI, CT, and brain mask images are loaded into the iElectrodes toolbox. Example images correspond to patient 2, implanted with an $8 \times 8$ grid over the left frontal, temporal, and parietal lobes.

\section{Masking and Thresholding}

CT voxel intensities that corresponded to electrodes were higher than most of the head tissue intensities, with the exception of dense bone within the skull. The normalized brain mask was dilated and/or eroded using a cubical nucleus of $3 \times$ $3 \times 3$ voxels, removing the unwanted CT high intensity signal from the skull. The number of erosion or dilation iterations of the brain mask was adjusted according to the subject and electrode array needs. Simultaneously, a voxel intensity threshold was set to 1,800 Hounsfield units and then adjusted manually to visualize the voxel clusters corresponding to the electrodes without brain tissue. If the threshold was too low, it was not possible to distinguish one electrode from its neighbors. If the threshold was too high, electrode voxels were not well defined. Figure 2 shows the effect of different thresholding values. The threshold values were adjusted for each electrode array, to account for differences in intensity levels. Spurious non-electrode voxels were manually removed if necessary, for example when the connection cable from one depth electrode crosses over a grid, or the removal of the metallic markers located between the first two contacts within some grids.

\section{Voxel Selection, Clustering, and Localization}

We used the selection drawing tool to select the electrode voxels for each electrode array in a $3 \mathrm{D}$ space (Figure $\mathbf{3 A}$ ). This procedure is facilitated by rotations, zoom, and pan in the $3 \mathrm{D}$ space plot. After selection, all electrode voxels ( $\mathrm{N}$ voxels) were clustered using a K-means algorithm (Hartigan and Wong, 1979; Figure 3B). In this step, $\mathrm{K}$ is the number of electrodes within each electrode array, which was obtained from implantation notes. Briefly, the K-means algorithm divides the $\mathrm{N}$ voxels into $\mathrm{K}$ clusters, whilst minimizing the within-cluster sum of squares. Then, the center of mass coordinate $\mathrm{L}_{k}$ of each electrode ( $\mathrm{k}$ $=1,2, \ldots, \mathrm{K})$ is calculated as a weighted average of all voxel coordinates inside each cluster, using the voxels signal intensities 

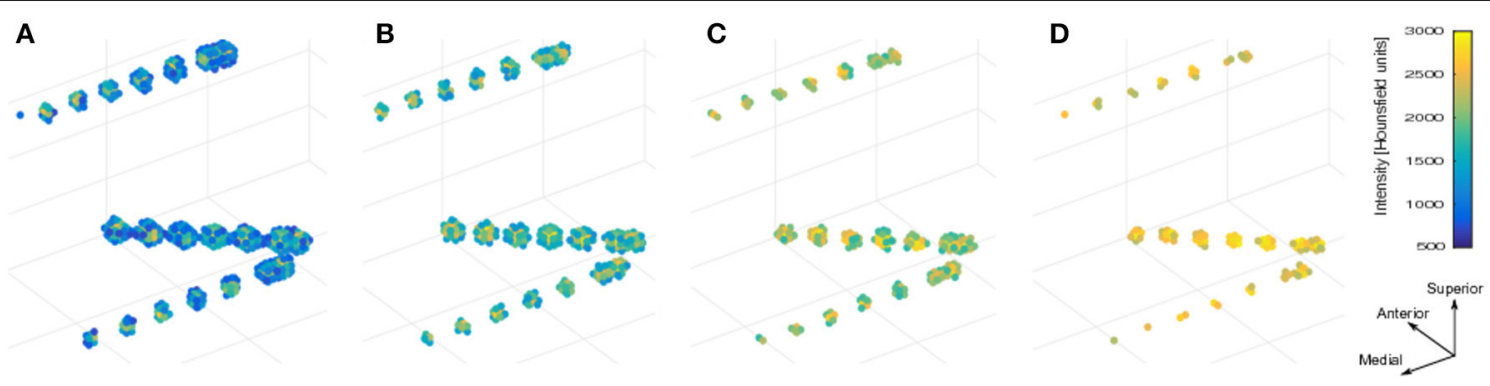

FIGURE 2 | Voxels thresholding. The use of different threshold values on the CT produces more diffuse or clear views of the electrodes. (A) A low threshold value $(700 \mathrm{HU})$ resulted in electrodes appearing to be merged together. (B,C) Middle threshold values $(1,200 \mathrm{HU}$ and 1,700 HU) resulted in a cleaner distinction between electrodes. (D) A high threshold value $(2,200 \mathrm{HU})$ resulted in a few voxels per electrode. Notice that the two medial (deeper) contacts in each array are closer $(3 \mathrm{~mm})$ than all other contacts, making its visual differentiation more difficult. Example data from patient 17 showing one frontal and two temporal depth electrode arrays. HU, Hounsfield Units.

as weights (Figure 3C). A matrix L, of dimension $\mathrm{Kx}$, was constructed containing all electrode coordinates.

\section{Automatic Electrode Numbering}

Each electrode contact had a different name or label assigned to it, uniquely identify the recording signal from a particular site. For every array of electrodes, we divided the labeling procedure into two steps; first numbering the electrodes and then naming. The numbering procedure was different for depth electrodes and grids. Here, we the terms numbering and indexing equivalently. The following steps are illustrated in Figures 3, 4.

- Depth electrodes.

(1) Since these electrode arrays are well described in a unidimensional space, we projected the $\mathrm{L}_{k}$ coordinates onto the first component (maximum variance dimension) of the Principal Component Analysis (PCA) decomposition of L.

(2) Electrodes were automatically numbered according to their ordinal position in this dimension. Number 1 was assigned to the deepest electrode.

- Grid electrodes.

(1) We projected the grid coordinates $\mathrm{L}_{k}$ onto the first two principal components (2D space) of the PCA decomposition of L (Figure 3D).

(2) A convex hull was calculated from these coordinates in the $2 \mathrm{D}$ space. We obtained a subset of $M$ points $P_{1}, P_{2}, \ldots, P_{M-1}$, $\mathrm{P}_{\mathrm{M}}$ describing the convex hull and $\mathrm{M}$ segments $\mathrm{S}_{1-2}, \mathrm{~S}_{2-3}$, ..., $\mathrm{S}_{\mathrm{M}-2-\mathrm{M}-1}, \mathrm{~S}_{\mathrm{M}-1-\mathrm{M}}$ connecting them (Figure 3D).

(3) We measured the angle at each segment transition, i.e., the angle between segments $S_{1-2}-S_{2-3}, S_{2-3}-S_{3-4}, \ldots$, $\mathrm{S}_{\mathrm{M}-2-\mathrm{M}-1}-\mathrm{S}_{\mathrm{M}-1-\mathrm{M}}, \mathrm{S}_{\mathrm{M}-1-\mathrm{M}}-\mathrm{S}_{\mathrm{M}-1}$ (Figure 3D). The grid corners were associated to the four largest transition angles, assuming that we were working on rectangular grids.

(4) An "ideal" grid was modeled using the corner coordinates, and the known number of rows and columns. This procedure estimated the expected coordinate of each electrode (Figure 3E). Corner electrodes in the real grid and ideal model correspond to the same point in space. We also specified electrode indexing numbers in the ideal array in accordance to its row and column position. Number 1 was assigned arbitrary to any of the possible corners coordinates.

(5) Real electrode coordinates lie in a curved surface, following the envelope of the brain contour (Figure 3C). Therefore, we radially projected the ideal grid coordinates to a spherical surface that best fits all real coordinates (Figure 3F).

(6) The electrode numbers were assigned to each real electrode according to the closest ideal electrode in terms of Euclidean distance (Figure 3G). Indices were assigned one by one, from short to long distances.

(7) The sum of distances between the real and ideal grids was minimized by permuting real grid indices (Figure 4):

(i) All neighbor electrode indices were permuted, one location at the time, by pairs in the row and column dimension of the grids. The sum of the distances among the real and ideal grid electrodes was measured at each permutation.

(ii) If one of these sum of distances was smaller than the nonpermuted one, the corresponding permutation was accepted and step (i) was repeated. If not, the loop was ended.

(8) The sum of distances between closest neighbors in the real grid was minimized following the same permutation procedure as in step 7 .

(9) Manual flips (up, down, left or right) and rotations (clockwise or counter-clockwise) of electrode numbers were done in agreement with real order of electrodes using implantation planning diagrams.

Naming electrodes was the last step for both grid and strips. Each electrode in the array was named using a prefix-number concatenation, for example Grid1, Grid2, Grid3,... GridK in a grid of $\mathrm{K}$ electrodes, or an ordered list of labels obtained from the iEEG recording files.

\section{Anatomical Labeling}

We used the Harvard-Oxford probabilistic atlas registered to the MNI152 space to assign anatomical labels to each electrode. This description is relevant to describe and compare activation areas across subjects. The atlas was obtained from the FMRIB Software Library v5.0 (FSL) (Jenkinson et al., 2012). 


\section{Thresholded voxels}

A

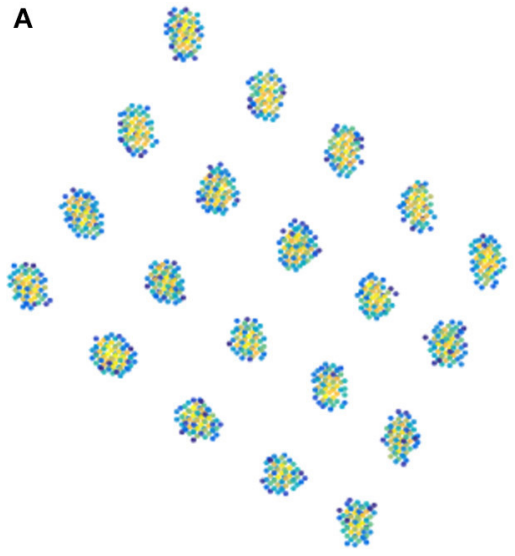

\section{Clustered voxels}

B

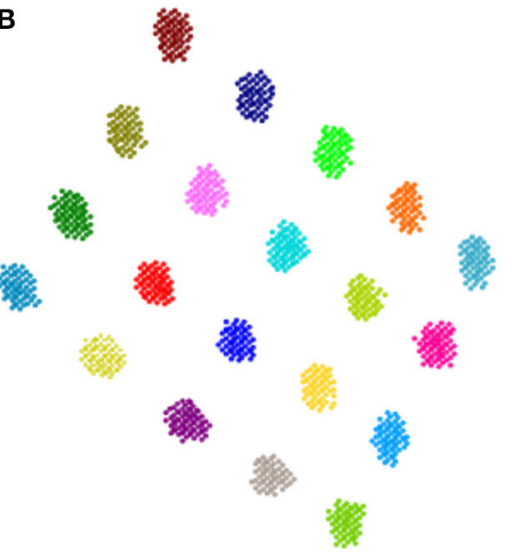

Ideal grid and real electrodes

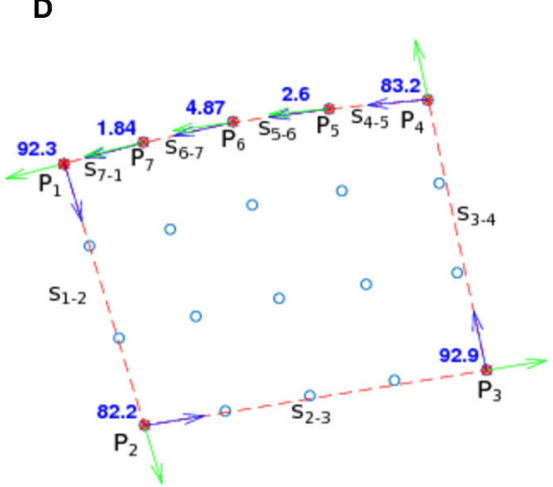

E

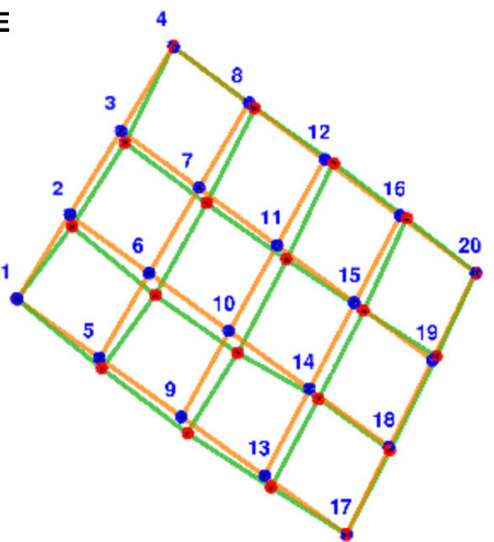

Electrode coordinates

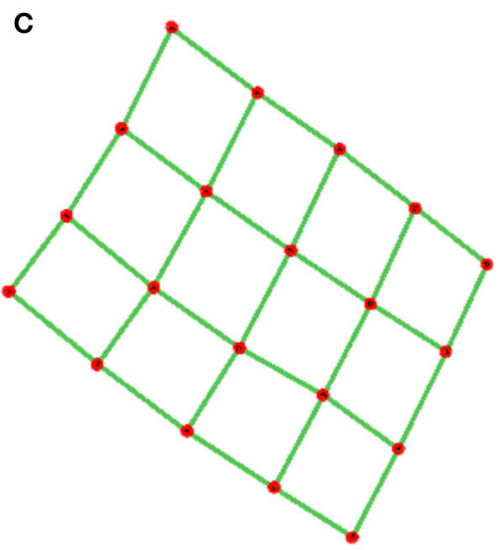

Spherically projected ideal grid and real electrodes
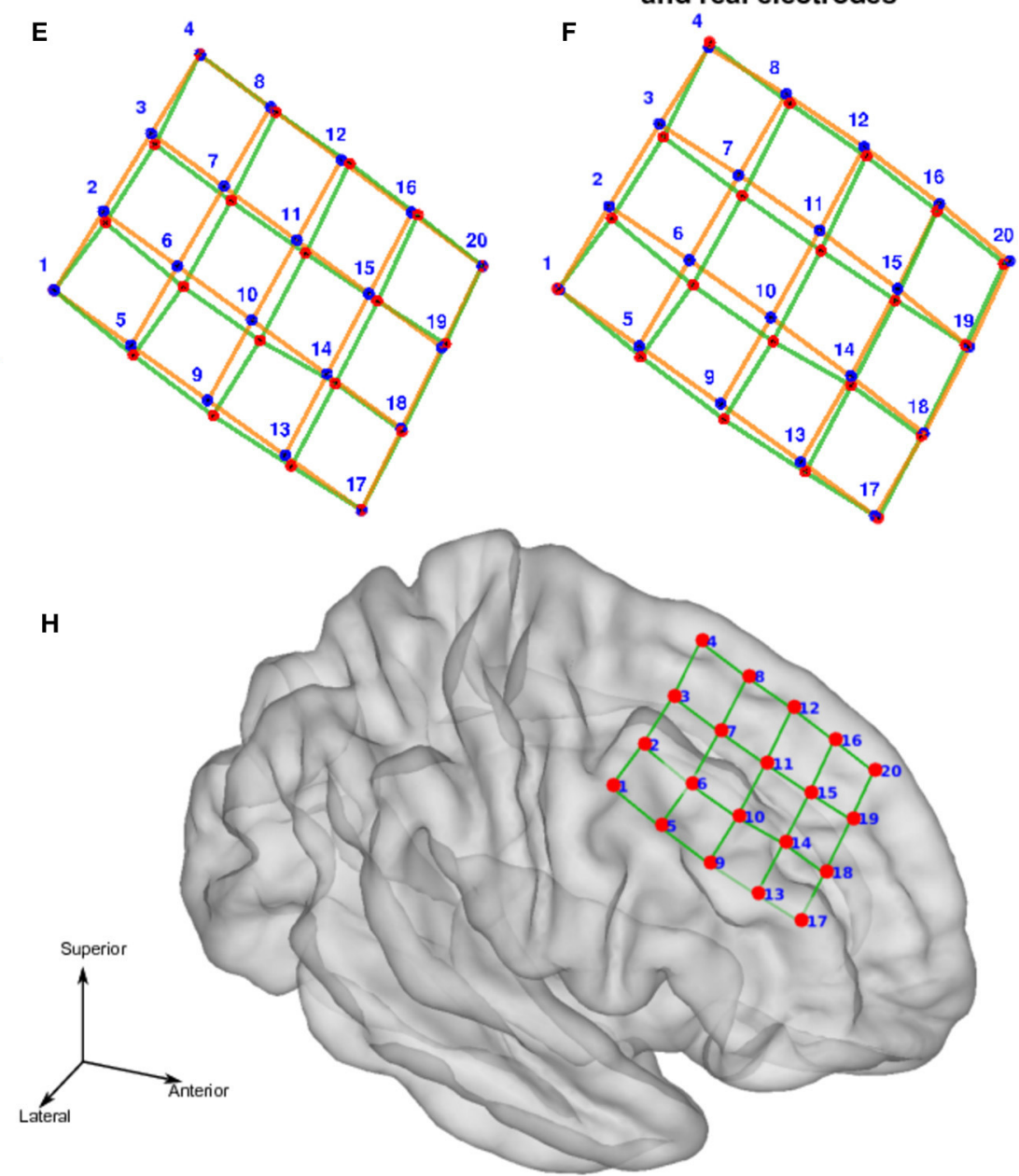

H

\section{Spherically projected ideal grid} and numbered real electrodes

G

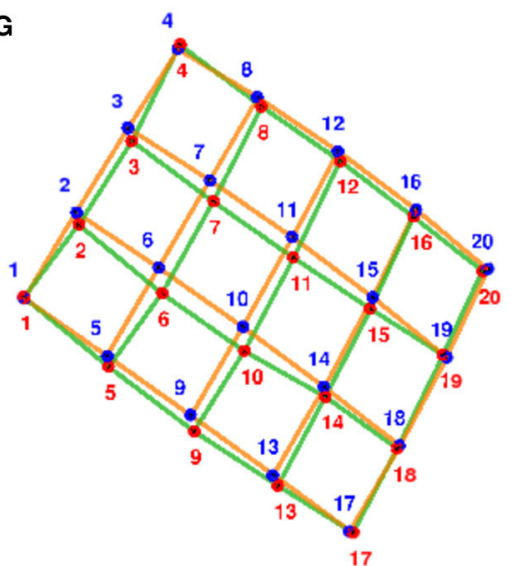



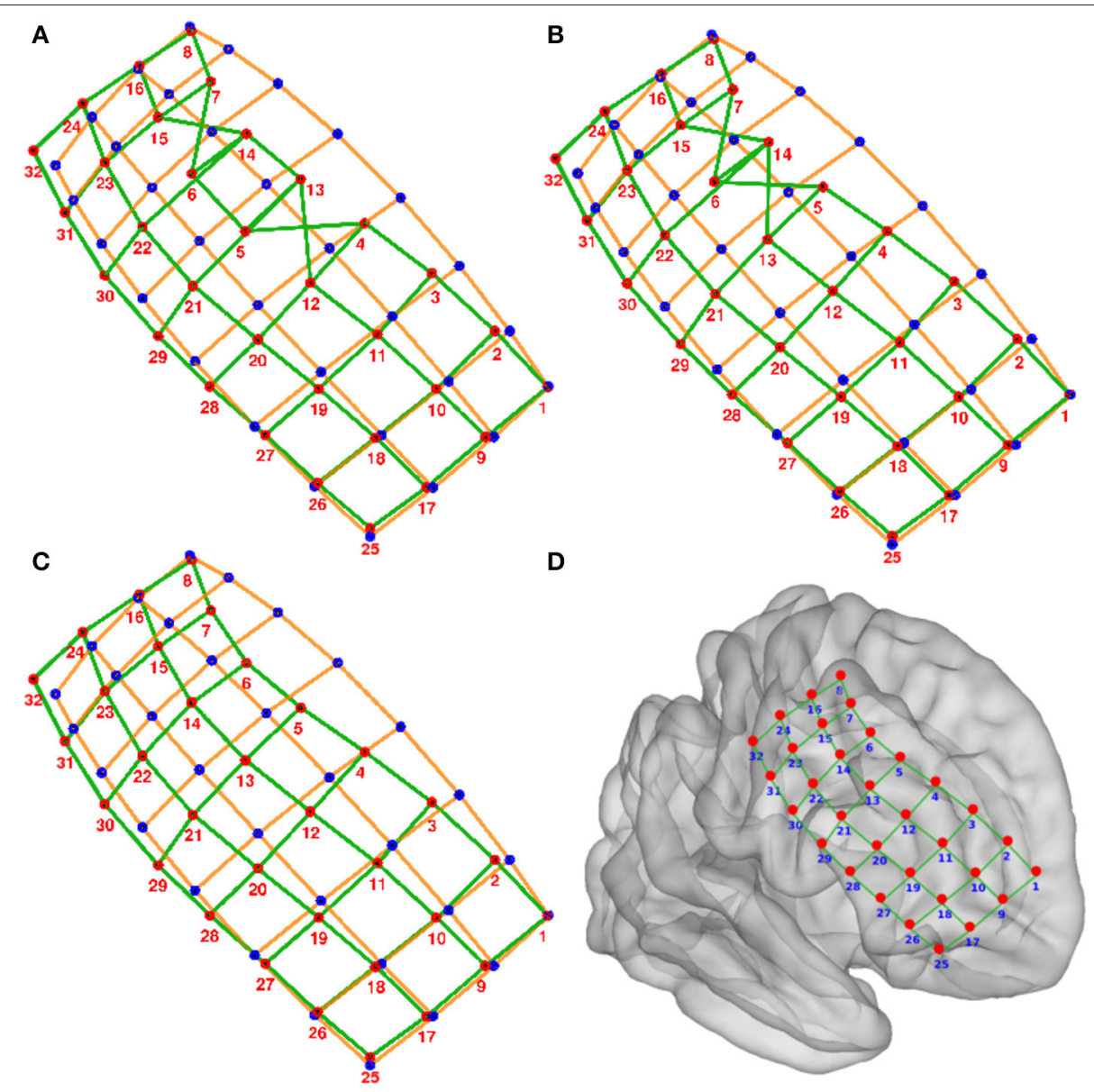

FIGURE 4 | Numbering of electrodes using permutation corrections. (A) First numbering of electrodes based on searching the minimum distance between ideal (blue dots) and real (red dots) electrodes. (B) Electrode numbering after first permutation correction. (C) Electrode numbering after second (last) permutation correction. (D) MNI semi-transparent brain with localized and numbered electrodes. Connecting lines are for illustrative purposes only. Example grid obtained from patient 3.

\section{Validation of Electrode Localization}

We defined three validation procedures to quantify the degree of success of the proposed method: visual inspection of position and labeling, quantitative measures of localization error, and normalization performance.

\section{Visual Validation}

Groups of electrodes in each array were visually checked in both $3 \mathrm{D}$ and $2 \mathrm{D}$ views. In $3 \mathrm{D}$ (Figures 3H, 4D) we checked that all electrodes were present and the labeling order was correct. Then we walked through all electrodes, one by one, and visually determined if the locations corresponded with the electrode artifact observed in the 2D orthogonal views of the fused MRI-CT images (Figures 5A-F).

\section{Quantitative Validation}

A group of five independent experts were asked to manually and semi-automatically localize a subset of electrodes to validate the proposed localization procedure. The experts had 3-10 years of experience in electrode localization utilizing neuroimaging methods. The subset of electrodes was randomly chosen from five patients, two with subdural grids and three with deep electrodes. A maximum of 32 electrodes were localized in the patients implanted with grid arrays. Two randomly chosen arrays were localized in each of the patients implanted with depth electrodes. This criterion was formulated to validate the method with a representative sample of electrodes.

In the manual localization procedure, the experts were instructed to determine the location of each electrode center based on the $2 \mathrm{D}$ visualization of $\mathrm{CT}$ artifacts, using the three orthogonal views of CT images. They set image brightness and contrast settings to their most comfortable values. They were able to select each coordinate, compare them, and correct their choices as many times as they wanted to. We will denote these manual locations as $L_{k, r}^{m}$ for electrode $\mathrm{k}$ and expert $\mathrm{r}$, being $\mathrm{k}=$ $1,2, \ldots, \mathrm{K}, \mathrm{r}=1,2, \ldots, \mathrm{R}, \mathrm{K}$ the total number of electrodes, and $\mathrm{R}$ the number of experts. Note that in order to simplify the notation, we have omitted the $x, y$, and $z$ components of each coordinate.

In the semi-automatic localization procedure, the group of experts localized the same subset of electrodes using 

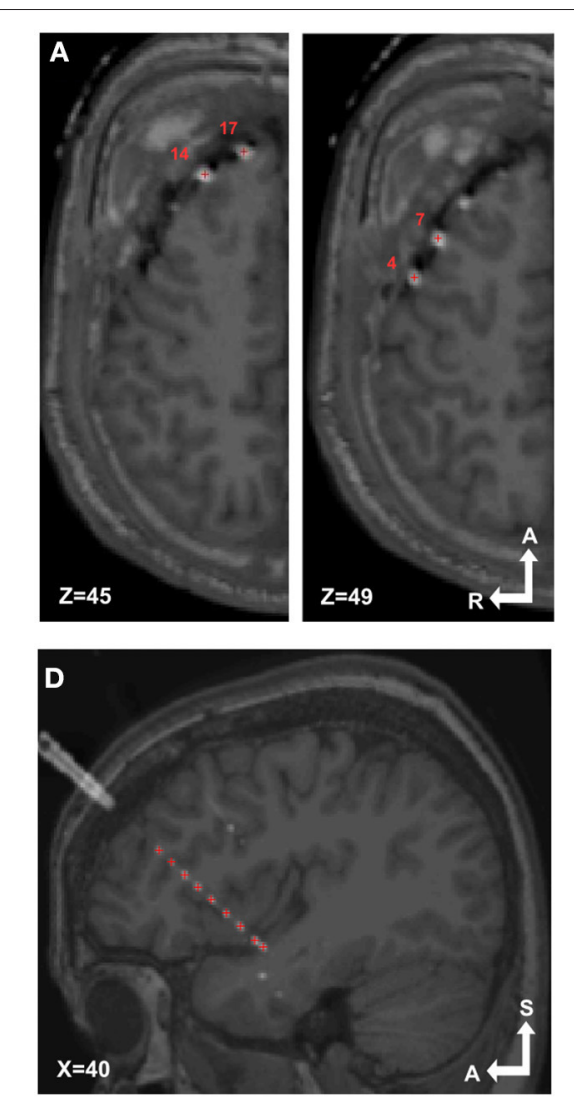
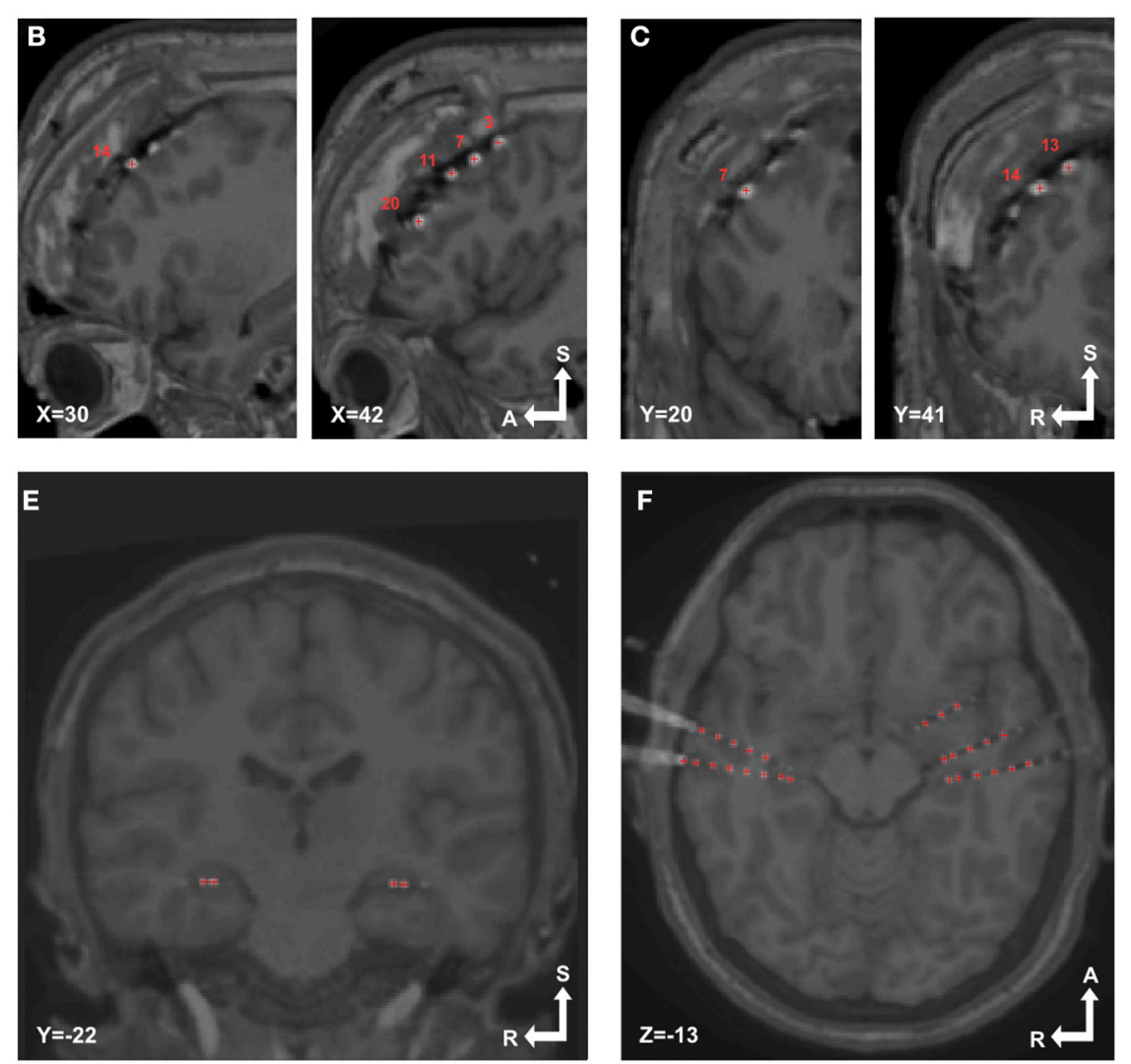

FIGURE 5 | Localized electrodes in MRI-CT. MRI-CT blend images showing examples of electrode artifacts and detected coordinates (red crosses) superimposed. (A-C) Axial, sagittal and coronal views (respectively) of patient 6, implanted with a $4 \times 5$ grid over the right frontal lobe. (D) Sagittal view of patient 14 showing a depth electrode array in the insular cortex. (E,F) Coronal and sagittal view of patient 18 showing bilaterally implanted electrodes in hippocampi and temporal lobes.

the iElectrodes toolbox as previously described in Section Localization of Intracranial Electrodes in iElectrodes Toolbox. We will denote these semi-automatic locations as $L_{k, r}^{s}$ for electrode $\mathrm{k}$ and expert $\mathrm{r}$.

Krippendorff's alpha (Krippendorff, 1970, 2004) was used as a measure of the inter-rater agreement of the localization procedures. Krippendorff's alpha has the capability to accept several raters and ordinal ratings (Hayes and Krippendorff, 2007). Alpha defines reliability scale points, being 1 for perfect reliability and 0 for the absence of reliability. Alpha values higher than 0.8 indicate that ratings are reliable (Krippendorff, 2004).

We defined the "gold standard" location $\mathrm{G}_{\mathrm{k}}$ for each electrode $\mathrm{k}$ as the mean of the manually localized coordinates across all experts $G_{k}=1 / R \sum_{r=1}^{R} L_{k, r}^{m}$.

Localization errors from using our semi-automatic method were calculated as the Euclidean distance of each electrode coordinate to the corresponding gold standard location $e_{k, r}^{s-G}=$ $\left\|L_{k, r}^{s}-G_{k}\right\|$, and equivalently for the manual method as $e_{k, r}^{m-G}=$ $\left\|L_{k, r}^{m}-G_{k}\right\|$ (Taimouri et al., 2014). Additionally, we used Krippendorff's alpha as a measure of the inter-rater agreement of the manual localization procedure, and therefore the reliability of the "gold standard."
Non-parametric permutation test statistics were used to evaluate the differences between semi-automatic and manual localization errors $\left(e_{k, r}^{s-G}\right.$ and $e_{k, r}^{m-G}$ respectively) (Nichols and Holmes, 2001). Localization errors for depth and grid electrodes were analyzed individually and combined together. This simple method does not depend on Gaussian distribution assumptions about the probability distribution of the data. The combined data from the manual and semi-automatic electrode locations underwent a random partition, and a $t$-test was calculated. This process was repeated 100,000 times to construct a $t$ value distribution under the null hypothesis of no difference between the two procedures. From the test statistic that was actually observed and the permutation distribution, we calculated the proportion of random partitions that resulted in a larger test statistic than the observed one. The resulting percentile is reported as a $p$-value (Maris and Oostenveld, 2007).

We assessed the robustness of the proposed method to thresholding, masking and voxel selection by the different experts. We calculated location $\mathrm{V}_{\mathrm{k}}$ for each electrode $\mathrm{k}$ as the mean of the semi-automatically localized coordinates across all experts $V_{k}=1 / R \sum_{r=1}^{R} L_{k, r}^{s}$. We measured the error distance between electrode locations obtained semi-automatically to their corresponding mean coordinate $e_{k, r}^{s-V}=\left\|L_{k, r}^{s}-V_{k}\right\|$. We 
quantified the error distribution by its mean and standard deviation. Additionally, we used Krippendorff's alpha as a measure of the inter-rater agreement of the semi-automatic localization procedure.

\section{Projection to Smoothed Cortical Envelope and Normalization Performance}

Subdural grids were expected to be on top of the cortical envelope of the MNI brain after the normalization procedure. Therefore, a smoothed cortical envelope (SCE) was constructed to take into account electrodes sitting above sulci. An MNI brain mask image was obtained from the Harvard-Oxford atlas in FSL (HarvardOxford-sub-maxprob-thr25-1mm.nii file) (Jenkinson et al., 2012). The brain stem structure and the cerebellum were removed, and left and right hemispheres were split. The image was processed with the vol2surf function ("cgalsurf" method) in ISO2Mesh toolbox for MATLAB (Fang and Boas, 2009). A tetrahedral mesh surface of the brain cortex was obtained for each hemisphere, the so called SCE.

Grid electrodes were projected to this surface while minimizing an energy cost function that considered the electrodes' displacement and the deformation of a spring like grid connecting the electrodes (Dykstra et al., 2012):

$$
\sum_{k=1}^{K}\left\|L_{k}-L_{k 0}\right\|^{2}+\sum_{i=1}^{K} \sum_{j=i+1}^{K} a_{i j}\left(d_{i j}-d_{i j 0}\right)^{2}
$$

constrained to $\forall k,\left\|L_{k}-s_{k}\right\|^{2}=0$, where $\mathrm{L}_{\mathrm{k}}$ is the coordinate of electrode $\mathrm{k}, \mathrm{L}_{\mathrm{k} 0}$ is the original coordinate for electrode $\mathrm{k}, \mathrm{d}_{\mathrm{ij}}$ is the distance between electrodes $i$ and $j, d_{i j 0}$ is the original distance among the same pair of electrodes, $\mathrm{a}_{\mathrm{ij}}$ is a parameter that take the value of 1 when electrodes $i$ and $j$ are neighbors and 0 otherwise, and $s_{\mathrm{k}}$ is the closest node in the SCE mesh to electrode $\mathrm{k}$. The minimization procedure was implemented in MATLAB using the "fmincon" function. The projection vector for each grid electrode is $\mathrm{D}_{\mathrm{k}}=\mathrm{L}_{\mathrm{k}}-\mathrm{L}_{\mathrm{k} 0}$.

When depth electrodes were implanted simultaneously to grids, a displacement field function was estimated based on the grid electrodes' projection (Taimouri et al., 2014). Translations were applied to the depth electrodes with variable strength according to their distance to the grid electrodes. We defined a weight function for each pair of grid electrode $k$ and depth electrode $\mathrm{j}$ as:

$$
w_{j k}=\exp \left(-\frac{\left\|L_{j}-L_{k}\right\|^{2}}{\sigma_{R}^{2}}\right)
$$

where $\sigma_{R}$ is a regularization parameter.

Depth electrodes close to the brain geometrical center were less affected by the spatial normalization than the ones on the brain surface. Accordingly, we calculated a weight function to attenuate the deformation field with distance

$$
w_{j k}^{\prime}=\exp \left(-\frac{\left\|L_{j}-L_{k}\right\|^{2}}{\sigma_{D}^{2}}\right)
$$

where $2 \sigma_{\mathrm{D}}$ was calculated as the mean distance of all grid electrodes to the center of mass of the SCE. Finally, the displacement vector for each depth electrode $\mathrm{j}$ was calculated as:

$$
F_{j}=\frac{\sum_{k=1}^{K} w_{j k} w_{j k}^{\prime} D_{k}}{\sum_{k=1}^{K} w_{j k}}
$$

Supplementary Figure 2 shows a simulated example of the displacement field function.

The performance of the normalization process was assessed by measuring the projection distance of grid electrodes to the SCE \| $\mathrm{D}_{k} \|$, and the displacement distance of depth electrodes $\left\|\mathrm{F}_{j}\right\|$.

\section{RESULTS}

We localized 1,242 electrodes (612 grid and 630 depth) in 22 patients using the proposed method of pre-processing and the toolbox (see Table 1 for electrodes locations details). Electrodes were distributed across all brain lobes in both hemispheres. Figure 6 shows the electrode positions from all patients superimposed over a MNI standard brain.

Some geometrical configurations of electrodes were more complex than others, such as when depth electrodes crossed very closely, when depth electrodes penetrated through grids, or when connection cables lay along electrode grids. In these cases, 3D views were especially useful in determining which $\mathrm{CT}$ voxels corresponded to each array. Figure 7 shows the localization results in $3 \mathrm{D}$ views for two scenarios, a simple case with only depth electrodes, and a more complex case, with two lateral grids and three depth electrodes entering through the gap between these two grids.

The time required for pre-processing was $\sim 7 \mathrm{~h}$ per participant, of which about 95 per cent was used to obtain the brain mask in FreeSurfer. The other processes took on average $15 \mathrm{~min}: \mathrm{MRI}$ and $\mathrm{CT}$ registration $\sim 5 \mathrm{~min}, \mathrm{MRI}$ and CT normalization $\sim 7 \mathrm{~min}$, MRI brain extraction $\sim 3 \mathrm{~min}$ (in a Dell Precision, 8 cores $2.3 \mathrm{GHz}, 16 \mathrm{~GB}$ RAM, Dell Inc. USA). Then, electrode localization and electrode labeling in iElectrodes toolbox required 2-3 min per electrode array.

Electrode coordinates were saved in MATLAB and text format files for future use with common EEG analyses toolboxes such as EEGLAB (Delorme and Makeig, 2004), FieldTrip (Oostenveld et al., 2011), and SPM (Litvak et al., 2011).

\section{Visual Validation}

During visual inspection, all 1,242 electrodes were located correctly over the corresponding CT artifact in the 2D orthogonal views of the blended MRI-CT images (see Figures $\mathbf{5 A - F}$ as an example). Additionally, we verified in the $3 \mathrm{D}$ rendered view the numbering of the electrodes.

\section{Quantitative Validation}

A group of 5 experts manually localized a subset of 91 electrodes in 5 patients (number 2, 7, 13, 17, and 21). The experts localized the electrodes both manually and using the toolbox. For 


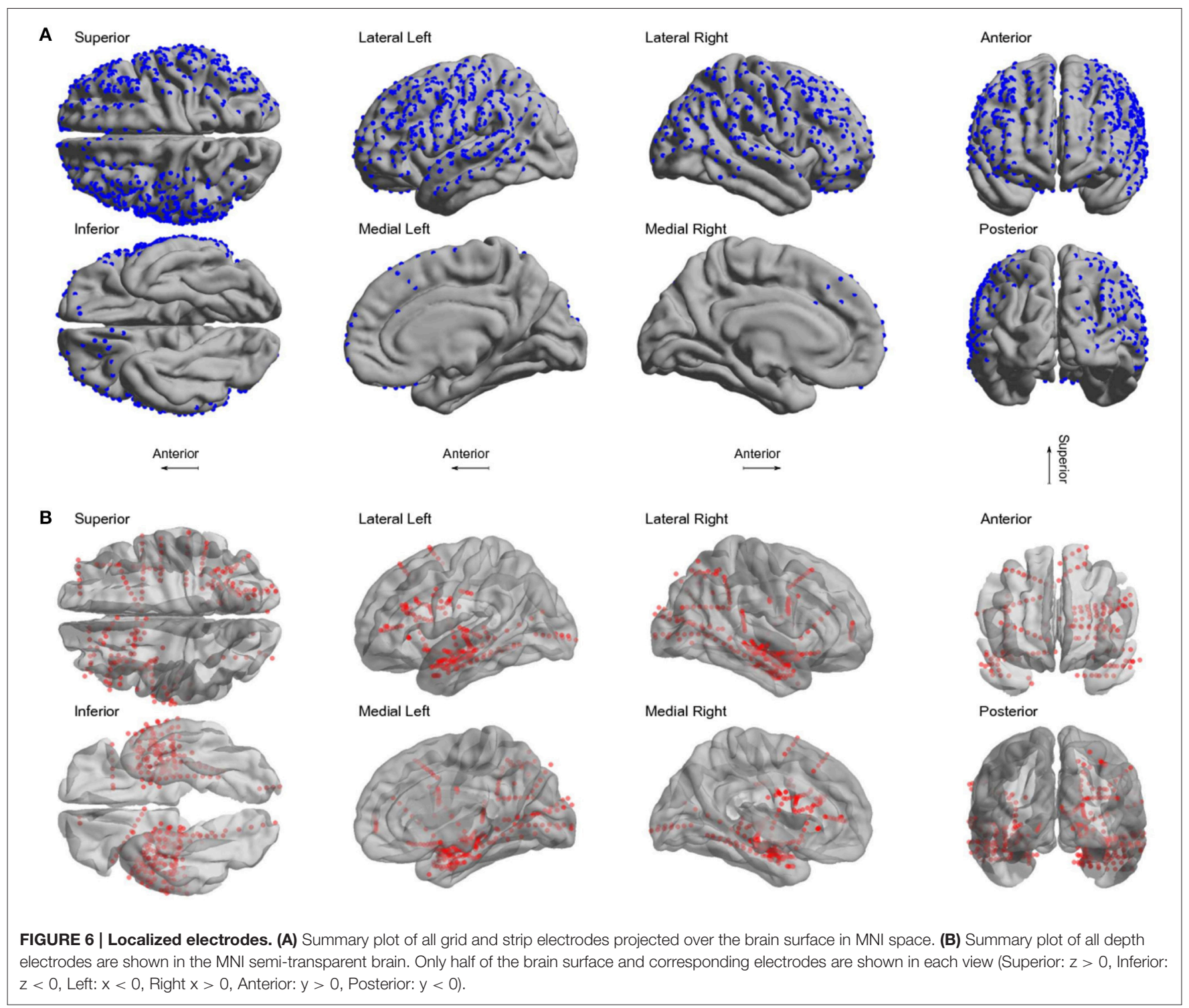

each electrode, the mean coordinate from the expert's manual localization was taken as the "gold standard" location to assess the accuracy of the new semi-automatic method as well as the manual method. Krippendorff's alpha, a measure of the inter-rater agreement, was 0.995 for the manual localization coordinates indicating that the experts coordinates were reliable to build a "gold standard." Overall, the mean localization distance per electrode between the gold standard and the new semiautomatic method was $\overline{e^{s-G}}=0.56 \mathrm{~mm} \pm 0.28 \mathrm{~mm}$, significantly smaller than the manual method $\overline{e^{m-G}}=0.79 \mathrm{~mm} \pm 0.38 \mathrm{~mm}$ $\left(p<1 \times 10^{-5}\right.$, permutation paired $t$-test). Also individually, for depth electrodes and grid electrodes, the proposed method achieved smaller localization errors than the manual method: for depth electrodes there was $\overline{e_{\text {depth }}^{s-G}}=0.46 \mathrm{~mm} \pm 0.18 \mathrm{~mm}$ vs. $e_{\text {depth }}^{m-G}$ $=0.71 \pm 0.21 \mathrm{~mm}\left(p<1 \times 10^{-5}\right.$, permutation paired $t$-test $)$, and for grid electrodes there was $\overline{e_{\text {grid }}^{s-G}}=0.63 \mathrm{~mm} \pm 0.11 \mathrm{~mm}$ vs. $\overline{e_{\text {grid }}^{m-G}}$
$=0.83 \mathrm{~mm} \pm 0.25 \mathrm{~mm}\left(p<1 \times 10^{-5}\right.$, permutation paired $t$-test $)$. Therefore, the localization using the semi-automatic method showed to be more accurate than the manual one in all cases. Figure 8 shows a box-whisker plot of the localization errors for each category.

The average time reported by experts for manual localization was $49 \mathrm{~min}$ (minimum 33, maximum $70 \mathrm{~min}$ ), whereas it took $22 \mathrm{~min}$ (minimum 20, maximum $25 \mathrm{~min}$ ) to operate the semiautomatic localization. It is important to notice that the manual localization time did not include numbering and anatomical labeling, which were automatically and almost instantaneously obtained with the proposed method.

We investigated the robustness of the proposed method, measuring the distance between the semi-automatic localized electrodes by the individual experts with its mean semiautomatic localization. The variability in localizations was due to thresholding, masking and voxel selection. The mean error 


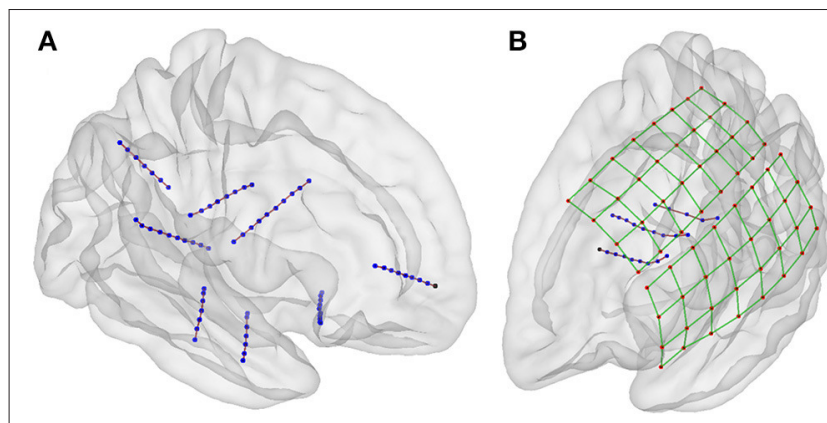

FIGURE 7 | Localization of electrodes in 3D view. (A) An example of localized of depth electrodes in a simple scenario, consisting of eight depth electrode arrays (Patient 22). (B) Example of localized of depth and grid electrodes in a complex scenario, formed by two grids and three depth electrode arrays entering through the gap between these two (Patient 5). In the last scenario, the selection of voxels in a 3D view is extremely simpler than the selection in $2 \mathrm{D}$ views. Additionally, the $3 \mathrm{D}$ view of localized coordinates on a rendered semitransparent brain helps to easily understand the relationship between electrodes and brain anatomy.

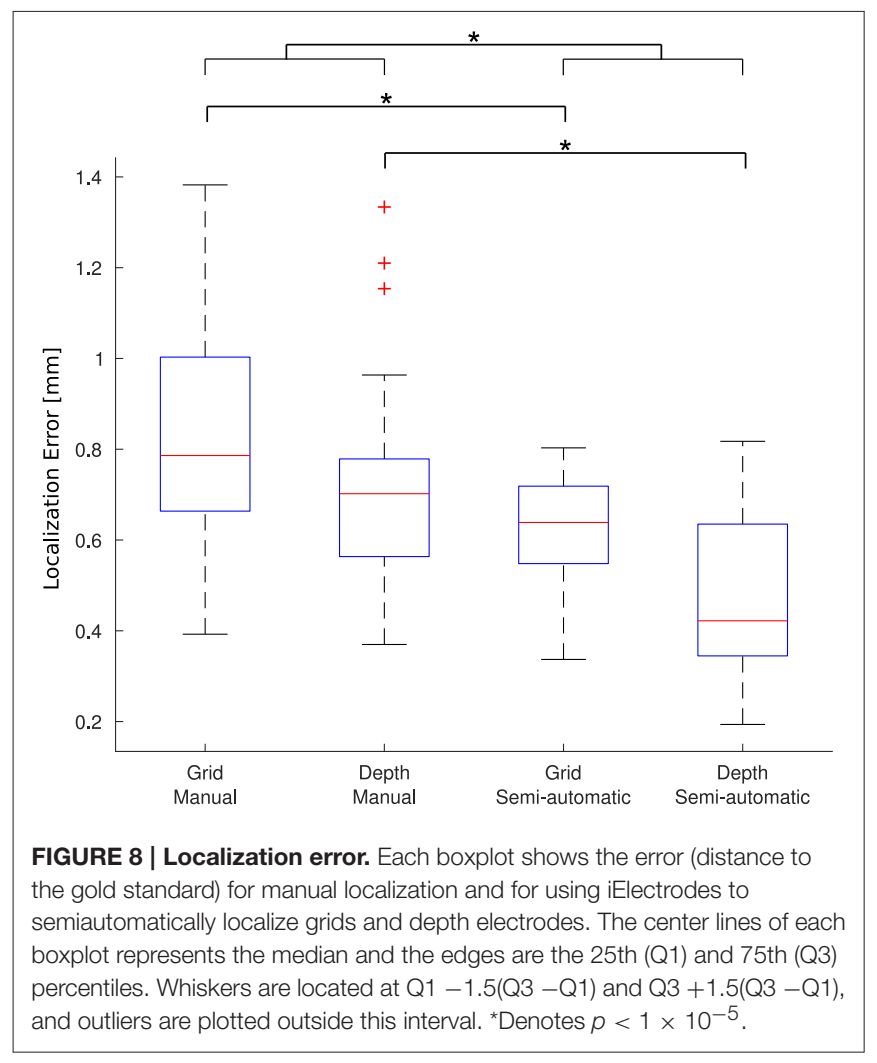

distance was $\overline{e^{s-V}}=0.10 \mathrm{~mm} \pm 0.05 \mathrm{~mm}$. Krippendorff's alpha was used as a measure of the inter-rater agreement of the proposed method. Krippendorff's alpha was 0.999 indicating nearly-perfect reliability (Krippendorff, 2004).

\section{Normalization Performance}

Grid electrodes were projected to the SCE while minimizing an energy cost function. The median displacement of grid electrodes $\left(\left\|\mathrm{D}_{k}\right\|\right)$ was $2.31 \mathrm{~mm}(\bar{D}=2.77 \mathrm{~mm} \pm 1.62 \mathrm{~mm})$ and maximum distance $7.46 \mathrm{~mm}$. When depth and grid electrodes were combined (patients 4-11), a displacement field function was estimated based on the grid electrodes' projection and applied to the depth electrodes. The median displacement of depth electrodes $\left.\left(\left\|\mathrm{F}_{j}\right\|\right)\right)$ was $0.56 \mathrm{~mm}(\overline{\|F\|}=0.81 \mathrm{~mm} \pm$ $0.81 \mathrm{~mm}$ ) and a maximum displacement of $3.21 \mathrm{~mm}$. Figure 9 shows the projection distance histograms for each and all patients implanted with grids. No displacement was calculated in patients implanted with deep electrodes only (patients 12-22).

To understand the variability of these distances, we tested and found no correlation between electrodes displacement and either the total number of implanted electrodes or the size of the grid (Spearman's rank correlation test, $p=0.97$ and $p=$ 0.93 respectively). However, we noticed a correlation between the electrodes distance to the SCE and the SCE distance to the pial brain surface (Spearman's rank correlation test, rho $=0.13, p=$ 0.001). In other words, the electrodes located over sulci showed a tendency of longer distances to the SCE (see Supplementary Figure 3).

\section{DISCUSSION}

We have introduced a new method to accurately and semiautomatically localize intracranial electrodes using MRI and CT images. The method returns electrode coordinate locations in standardized MNI space and electrode numbering. The evaluation included 22 patients implanted with a total of 1,242 electrodes, demonstrating that the method is robust with respect to electrode types, diverse implantation configurations, and anatomical regions.

A graphical user interface, iElectrodes, was developed to implement this method as an open-source application. The application allows $2 \mathrm{D}$ and $3 \mathrm{D}$ views on a rendered semitransparent brain that facilitates a straightforward understanding of electrodes locations in relationship to brain anatomy. The application also provides automatic anatomical labeling by utilizing atlases such as the Harvard-Oxford Structural atlas (Jenkinson et al., 2012). Thus, users are given the neuronal location of the electrodes, which is especially useful for neuroanatomical reference. Since the toolbox is open-source, other atlases can be easily imported. Moreover, parcellation images according to the Desikan-Killiany atlas (Desikan et al., 2006) and obtained from the FreeSurfer processing pipeline are supported by the toolbox. Desikan-Killiany parcellation from pre-implantation images showed to be particular useful for native space analyses in the clinical practice (Princich et al., 2013).

This new method of calculating electrode coordinates from the normalized CT images reduces the time it takes to manually identify electrode locations from MRI and/or CT images. Previous studies show that manual identification at hospitals can take anything from $6 \mathrm{~h}$ to 3 days (Princich et al., 2013) and requires an expert in MRI neuroanatomy. In comparison, methods like photograph localization can take 3-5 h (Dalal et al., 2008; Pieters et al., 2013). Automatic localization procedures 

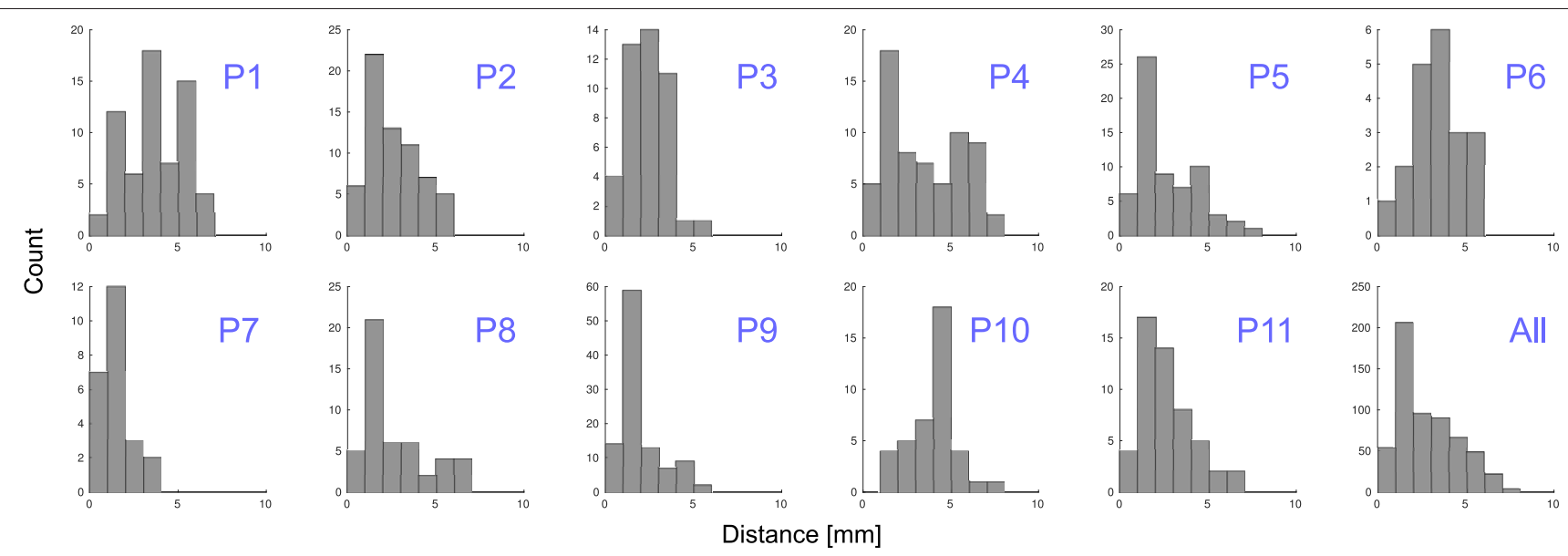

FIGURE 9 | Distance to MNI cortex. Histograms showing the estimated distance of subdural grid electrodes to the smoothed cortical envelope (SCE) for each patient, P1-P11, and for all of them together.

have been proposed reducing the processing time to $15 \mathrm{~min}$ (Sebastiano et al., 2006; Taimouri et al., 2014; Arnulfo et al., 2015). However, these earlier automatic methods are limited. Arnulfo et al. (2015) described a method restricted solely to depth electrodes which requires stereotactic implantation coordinates. Sebastiano et al. (2006) presented an automatic method using spatial filters, but is restricted to detect grid type electrodes only. Taimouri et al. (2014) extended the idea to grid and depth electrodes coordinates, yet the method fails to automatically localize all depth electrodes or distinguish close contacts. Alternatively, Yang et al. (2012) proposed a method based on the selection of only a few electrodes, reducing localization time. However, the technique is limited to grids without curvature. Given the limitations of these alternative methods and even though our proposed method is not fully automatic, it substantially reduces localization time to a few minutes and precisely localizes all implanted depth and subdural grid electrodes. Furthermore, we provided a method to automatically number depth and grid electrode arrays.

Our proposed method relies on the detection of CT signals, which are often used for the localization of electrodes in epilepsy intracranial recordings (Ekstrom et al., 2008; Princich et al., 2013; Arnulfo et al., 2015) and in deep brain stimulation (Hebb and Poliakov, 2009; Lee et al., 2010; Horn and Kühn, 2015). Thus, the manual localization of CT artifacts was considered a gold standard in this study. LaViolette et al. (2011b) validated artifact detection in post-implantation MRI and CT coregistrations and showed a qualitative correspondence with intraoperative images; however they reported differences due to brain compression in the CT and MRI images.

Electrode location inaccuracy may occur using observational methods because the central coordinate of each electrode is subjectively identified from a CT image. Instead, we offer a simple method that identifies the central coordinate by calculating the voxel cluster's center of mass. Krippendorff's alpha and a small distance error $\left(\overline{e^{s-V}}\right)$ showed that our method is robust to user variations including: a wide range of thresholding values, different numbers of mask erosion or dilation iterations, and electrode voxel selections. Also, it has higher accuracy than the manual localizations done by experts $\left(\overline{e^{s-G}}\right.$ was significantly smaller than $\overline{e^{m-G}}$ ).

The accuracy achieved by our method was $0.56 \mathrm{~mm}$ in contrast to the gold standard manual localization. Similarly, Arnulfo et al. (2015) showed localization errors of $0.5 \mathrm{~mm}$ for depth electrodes using an automated method for detecting CT artifacts. In comparison, for subdural grids, methods coregistering intraoperative photography, MRI and X-ray images showed larger localization errors of $\sim 2 \mathrm{~mm}$ (Dalal et al., 2008). Previous methods projecting post-implantation CT coordinates onto pre-implantation MRIs reach mean localization errors in the range of 1.31-3.04 mm (Hermes et al., 2010; Dykstra et al., 2012; Yang et al., 2012; Taimouri et al., 2014). In these cases, the CT is known to be deformed while the MRI is not and localized grid electrodes appear "buried" in the cortex up to $14 \mathrm{~mm}$ due to fluid build-up (Dalal et al., 2008; Dykstra et al., 2012). The accumulation of fluid is a dynamic process that occurs while electrodes are implanted in the brain (Studholme et al., 2001; LaViolette et al., 2011a,b). Another factor effecting localization is the brain displacement during surgical procedures, when intraoperative photographs are taken, which can exceed $10 \mathrm{~mm}$ with the dominant directional component being associated with gravity, and the mean displacement independent of the size and orientation of cranial opening (Roberts et al., 1998). Moreover, LaViolette et al. (2011a) showed grid electrode mean displacements of $5.4 \mathrm{~mm}$ (shear of $4.0 \mathrm{~mm}$ ) between implantation surgery and cranial reopening. Accordingly, there is no clear evidence that intra-operative photography is more reliable than post-implantation images for localization purposes. Both techniques provide clear localization evidence at the time they are performed, but changes may occur since brain deformation is a dynamic process (Studholme et al., 2001). Additionally, post-implantation images have the advantage of being acquired closer in time to the cognitive task recordings. 
When using post-implantation MRI and CT, even though both images account for the same levels of deformation, their coregistration becomes a source of errors (Azarion et al., 2014). Therefore, we applied a maximization of mutual information technique, which has been widely used for cross modality coregistrations (Pluim et al., 2003; LaViolette et al., 2011b; Azarion et al., 2014) and showed sub-voxel accuracy results (Maes et al., 1997).

We have emphasized the potential applications of iEEG to cognitive neuroscience, for which iElectrodes provides a tool to compare recording sites with non-invasive studies and population level analysis. We consider the normalization performance achieved by our method as sufficient for identifying the cortical activations in most cognitive neuroscience research studies, but it could, nevertheless, be subject to improvements (median projection displacement $\left\|\mathrm{D}_{k}\right\|$ was $2.31 \mathrm{~mm}$ for grids electrodes, and median $\left\|\mathrm{F}_{j}\right\|$ was $0.56 \mathrm{~mm}$ for depths electrodes). Previously, we showed the relevance of using standardized brain spaces such as MNI to allow comparisons of invasive event related potentials with EEG group analysis (Chennu et al., 2013), or to show convergent evidence of a hierarchical prediction network arising from intracranial and MEG recordings (Phillips et al., 2016). Recently, Kadipasaoglu et al. (2014) demonstrated a new method for group analysis, using a surface-based analysis for iEEG grid recordings but it was not suitable for depth or combined depth and grid recordings. Our localization pipeline, could be combined with this approach to address their limitation. Where peri-operative distortions are large, other methods for normalization may have an advantage (see Klein et al., 2009, for a comparative evaluation of methods) and iElectrodes is compatible with other pre-processing methods such as Advance Normalization Tools (ANTS)-Inside Toolkit (ITK) (Avants et al., 2014) and SPM DARTEL (Ashburner, 2007). We recommend iElectrodes users to utilize the best registration and normalization tools available, and to quantify the pre-processing errors when possible.

Even though several studies showed the safety of postimplantation MRI (Davis et al., 1999; Carmichael et al., 2008) some hospitals do not perform this imaging technique with implanted electrodes. To overcome this limitation we provide the users of our toolbox with the possibility of projecting grids to the SCE surface using the method developed by Dykstra et al. (2012) and then translating the depth electrodes accordingly (Taimouri et al., 2014). It is also possible to use the proposed localization pipeline in the native patient space without normalization. However, we did not test native space localization and cannot comment on whether errors and reliability of the method are different without normalization.

In summary, we provide a new toolbox for the research community that meets the criteria of accurate electrode localization of depth and grid electrodes, offers simplicity and speed of application, reliability, and accessible open-source software.

\section{AVAILABILITY AND COMPATIBILITY WITH OTHER TOOLBOXES}

The software project is made available to the scientific community in the form of an open-source MATLAB ${ }^{\circledR}$ toolbox that can be freely downloaded from https://sourceforge.net/projects/ielectrodes/ for research porpoises only under GNU General Public License. We recommend iElectrodes users to run on MATLAB version 2016a or latter. Due to technical limitations in MATLAB's graphical engine, some minor visual failures were observed in older versions. However, we will provide compatibility up to version 2013a for a limited period of time. Additional code is provided in order to facilitate pre-processing and interaction with electro-physiology analysis toolboxes as EEGLAB (Delorme and Makeig, 2004) and FieldTrip (Oostenveld et al., 2011).

\section{AUTHOR CONTRIBUTIONS}

$A B$ wrote the article. $A B$ designed and developed the software. $\mathrm{AB}$ and $\mathrm{HP}$ contributed to the code. JP acquired the images. $\mathrm{AB}$ and HP analyzed the data. JR, TB, CM, and SK supervised this work and wrote the article.

\section{FUNDING}

This work was supported by Consejo Nacional de Investigaciones Científicas y Técnicas (CONICET) to AB and SK, Agencia Nacional de Promoción Científica y Tecnológica (PIDC 53/2012 and PICT 0775/2012 to AB, JP, SK, and PICT 1232/2014 to CM), Universidad Nacional Arturo Jauretche Investiga 2014 to $\mathrm{AB}$ and $\mathrm{SK}$, Comisión de Investigaciones Científicas (CIC) to CHM, Medical Research Council (MC-A060-5PQ30 to JR and a Doctoral Training award to HP), Wellcome Trust (103838 Senior Research Fellowship to JR, Biomedical Research Fellowship; WT093811MA to TB), the James F. McDonnell Foundation 21st Century Science Initiative: Understanding Human Cognition to JR.

\section{ACKNOWLEDGMENTS}

The iElectrodes toolbox uses subroutines authored by Jimmy Shen ("Tools for NIfTI and ANALYZE image"), Qianqian Fang and David Boas ("iso2mesh"), Levente Hunyadi ("spherefit"), and Divahar Jayaraman ("Cylinder") that are covered by a BSDlicense allowing modification and re-distribution. The authors would like to thank James Lubell, Santiago Collavini and Simon Jones for manually and semi-automatically localizing the electrodes for validation.

\section{SUPPLEMENTARY MATERIAL}

The Supplementary Material for this article can be found online at: http://journal.frontiersin.org/article/10.3389/fninf. 2017.00014/full\#supplementary-material 


\section{REFERENCES}

Arnulfo, G., Narizzano, M., Cardinale, F., Fato, M. M., and Palva, J. M. (2015). Automatic segmentation of deep intracerebral electrodes in computed tomography scans. BMC Bioinformatics 16:99. doi: 10.1186/s12859-015-0511-6

Ashburner, J. (2007). A fast diffeomorphic image registration algorithm. Neuroimage 38, 95-113. doi: 10.1016/j.neuroimage.2007.07.007

Ashburner, J., and Friston, K. J. (1999). Nonlinear spatial normalization using basis functions. Hum. Brain Mapp. 7, 254-266.

Avants, B. B., Tustison, N. J., Stauffer, M., Song, G., Wu, B., and Gee, J. C. (2014). The Insight ToolKit image registration framework. Front. Neuroinform. 8:44. doi: 10.3389/fninf.2014.00044

Axmacher, N., Cohen, M. X., Fell, J., Haupt, S., Dümpelmann, M., Elger, C. E., et al. (2010). Intracranial EEG correlates of expectancy and memory formation in the human hippocampus and nucleus accumbens. Neuron 65, 541-549. doi: 10.1016/j.neuron.2010.02.006

Azarion, A. A., Wu, J., Pearce, A., Krish, V. T., Wagenaar, J., Chen, W., et al. (2014). An open-source automated platform for three-dimensional visualization of subdural electrodes using CT-MRI coregistration. Epilepsia 55, 2028-2037. doi: $10.1111 /$ epi.12827

Ball, T., Kern, M., Mutschler, I., Aertsen, A., and Schulze-Bonhage, A. (2009). Signal quality of simultaneously recorded invasive and non-invasive EEG. Neuroimage 46, 708-716. doi: 10.1016/j.neuroimage.2009.02.028

Bootsveld, K., Träber, F., Kaiser, W. A., Layer, G., Elger, C. E., Hufnagel, A., et al. (1994). Localisation of intracranial EEG electrodes using three dimensional surface reconstructions of the brain. Eur. Radiol. 4, 52-56. doi: 10.1007/BF00177388

Carmichael, D. W., Thornton, J. S., Rodionov, R., Thornton, R., McEvoy, A., Allen, P. J., et al. (2008). Safety of localizing epilepsy monitoring intracranial electroencephalograph electrodes using MRI: radiofrequency-induced heating. J. Magn. Reson. Imaging 28, 1233-1244. doi: 10.1002/jmri.21583

Chauvel, P., Buser, P., Badier, J. M., Liegeois-Chauvel, C., Marquis, P., and Bancaud, J. (1987). The "epileptogenic zone" in humans: representation of intercritical events by spatio-temporal maps. Rev. Neurol. 143, 443-450.

Chennu, S., Noreika, V., Gueorguiev, D., Blenkmann, A., Kochen, S., Ibáñez, A., et al. (2013). Expectation and attention in hierarchical auditory prediction. J. Neurosci. 33, 11194-11205. doi: 10.1523/JNEUROSCI.0114-13.2013

Dalal, S. S., Edwards, E., Kirsch, H. E., Barbaro, N. M., Knight, R. T., and Nagarajan, S. S. (2008). Localization of neurosurgically implanted electrodes via photograph-MRI-radiograph coregistration. J. Neurosci. Methods 174, 106-115. doi: 10.1016/j.jneumeth.2008.06.028

Dale, A. M., Fischl, B., and Sereno, M. I. (1999). Cortical surface-based analysis. I. Segmentation and surface reconstruction. Neuroimage 9, 179-194. doi: 10.1006/nimg.1998.0395

Dale, A. M., and Sereno, M. I. (1993). Improved localizadon of cortical activity by combining EEG and MEG with MRI cortical surface reconstruction: a linear approach. J. Cogn. Neurosci. 5, 162-176. doi: 10.1162/jocn.1993.5.2.162

Davis, L. M., Spencer, D. D., Spencer, S. S., and Bronen, R. A. (1999). MR imaging of implanted depth and subdural electrodes: is it safe? Epilepsy Res. 35, 95-98. doi: 10.1016/S0920-1211(99)00007-8

Delorme, A., and Makeig, S. (2004). EEGLAB: an open source toolbox for analysis of single-trial EEG dynamics including independent component analysis. J. Neurosci. Methods 134, 9-21. doi: 10.1016/j.jneumeth.2003.10.009

Desikan, R. S., Ségonne, F., Fischl, B., Quinn, B. T., Dickerson, B. C., Blacker, D., et al. (2006). An automated labeling system for subdividing the human cerebral cortex on MRI scans into gyral based regions of interest. Neuroimage 31, 968-980. doi: 10.1016/j.neuroimage.2006.01.021

Dykstra, A. R., Chan, A. M., Quinn, B. T., Zepeda, R., Keller, C. J., Cormier, J., et al. (2012). Individualized localization and cortical surfacebased registration of intracranial electrodes. Neuroimage 59, 3563-3570. doi: 10.1016/j.neuroimage.2011.11.046

Ekstrom, A., Suthana, N., Behnke, E., Salamon, N., Bookheimer, S., and Fried, I. (2008). High-resolution depth electrode localization and imaging in patients with pharmacologically intractable epilepsy. J. Neurosurg. 108, 812-815. doi: $10.3171 / \mathrm{JNS} / 2008 / 108 / 4 / 0812$

Elias, W. J., Fu, K.-M., and Frysinger, R. C. (2007). Cortical and subcortical brain shift during stereotactic procedures. J. Neurosurg. 107, 983-988. doi: $10.3171 /$ JNS-07/11/0983
Enatsu, R., Bulacio, J., Najm, I., Wyllie, E., So, N. K., Nair, D. R., et al. (2014). Combining stereo-electroencephalography and subdural electrodes in the diagnosis and treatment of medically intractable epilepsy. J. Clin. Neurosci. 21, 1441-1445. doi: 10.1016/j.jocn.2013.12.014

Fang, Q., and Boas, D. A. (2009). "Tetrahedral mesh generation from volumetric binary and grayscale images," in Proceedings - 2009 IEEE International Symposium on Biomedical Imaging: From Nano to Macro, ISBI 2009 (Boston, MA: IEEE), 1142-1145. doi: 10.1109/ISBI.2009.5193259

Fischl, B., Van der Kouwe, A., Destrieux, C., Halgren, E., Ségonne, F., Salat, D. H., et al. (2004). Automatically parcellating the human cerebral cortex. Cereb. Cortex 14, 11-22. doi: 10.1093/cercor/ bhg087

Gaillard, R., Dehaene, S., Adam, C., Clémenceau, S., Hasboun, D., Baulac, M., et al. (2009). Converging intracranial markers of conscious access. PLoS Biol. 7:e1000061. doi: 10.1371/journal.pbio.1000061

Gonzalez-Martinez, J., Bulacio, J., Alexopoulos, A., Jehi, L., Bingaman, W., and Najm, I. (2013). Stereoelectroencephalography in the "difficult to localize" refractory focal epilepsy: early experience from a North American epilepsy center. Epilepsia 54, 323-330. doi: 10.1111/j.1528-1167.2012.0 3672.x

Hartigan, J. A., and Wong, M. A. (1979). Algorithm AS 136: a K-means clustering algorithm. Appl. Stat. 28, 100. doi: 10.2307/2346830

Hayes, A. F., and Krippendorff, K. (2007). Answering the call for a standard reliability measure for coding data. Commun. Methods Meas. 1, 77-89. doi: 10.1080/19312450709336664

Hebb, A. O., and Poliakov, A. V. (2009). Imaging of deep brain stimulation leads using extended hounsfield unit CT. Stereotact. Funct. Neurosurg. 87, 155-160. doi: 10.1159/000209296

Hermes, D., Miller, K. J., Noordmans, H. J., Vansteensel, M. J., and Ramsey, N. F. (2010). Automated electrocorticographic electrode localization on individually rendered brain surfaces. J. Neurosci. Methods 185, 293-298. doi: 10.1016/j.jneumeth.2009.10.005

Horn, A., and Kühn, A. A. (2015). Lead-DBS: a toolbox for deep brain stimulation electrode localizations and visualizations. Neuroimage 107, 127-135. doi: 10.1016/j.neuroimage.2014.12.002

Ibáñez, A., Cardona, J. F., Dos Santos, Y. V., Blenkmann, A., Aravena, P., Roca, M., et al. (2013). Motor-language coupling: direct evidence from early Parkinson's disease and intracranial cortical recordings. Cortex 49, 968-984. doi: 10.1016/j.cortex.2012.02.014

Jenkinson, M., Beckmann, C. F., Behrens, T. E., Woolrich, M. W., and Smith, S. M. (2012). FSL. Neuroimage 62, 782-790. doi: 10.1016/j.neuroimage.2011. 09.015

Jenkinson, M., Pechaud, M., and Smith, S. (2005). "BET2 - MR-based estimation of brain, skull and scalp surfaces," in Eleventh Annual Meeting of the Organization for Human Brain Mapping (Toronto), 167.

Kadipasaoglu, C. M., Baboyan, V. G., Conner, C. R., Chen, G., Saad, Z. S., and Tandon, N. (2014). Surface-based mixed effects multilevel analysis of grouped human electrocorticography. Neuroimage 101, 215-224. doi: 10.1016/j.neuroimage.2014.07.006

Keller, C. J., Bickel, S., Entz, L., Ulbert, I., Milham, M. P., Kelly, C., et al. (2011). Intrinsic functional architecture predicts electrically evoked responses in the human brain. Proc. Natl. Acad. Sci. U.S.A. 108, 10308-10313. doi: 10.1073/pnas.1019750108

Ken, S., Di Gennaro, G., Giulietti, G., Sebastiano, F., De Carli, D., Garreffa, G., et al. (2007). Quantitative evaluation for brain CT/MRI coregistration based on maximization of mutual information in patients with focal epilepsy investigated with subdural electrodes. Magn. Reson. Imaging 25, 883-888. doi: 10.1016/j.mri.2007.02.003

Klein, A., Andersson, J., Ardekani, B. A., Ashburner, J., Avants, B., Chiang, M. C., et al. (2009). Evaluation of 14 nonlinear deformation algorithms applied to human brain MRI registration. Neuroimage 46, 786-802. doi: 10.1016/j.neuroimage.2008.12.037

Kochen, S., Giagante, B., Consalvo, D., Oddo, S., Silva, W., Solis, P., et al. (2002). Análisis retrospectivo (1984-2000). Experiencia en pacientes candidatos a cirugía de la epilepsia, (Buenos Aires, Argentina). Rev. Neurol. Argent. 27, $41-44$.

Kovalev, D., Spreer, J., Honegger, J., Zentner, J., Schulze-Bonhage, A., and Huppertz, H.-J. (2005). Rapid and fully automated visualization of subdural 
electrodes in the presurgical evaluation of epilepsy patients. AJNR. Am. J. Neuroradiol. 26, 1078-1083.

Krippendorff, K. (1970). Estimating the reliability, systematic error and rondom error of interval data. Educ. Psychol. Meas. 30, 61-70. doi: $10.1177 / 001316447003000105$

Krippendorff, K. (2004). Reliability in content analysis: some common misconceptions and recommendations. Hum. Commun. Res. 30, 411-433. doi: 10.1111/j.1468-2958.2004.tb00738.x

Kwan, P., Arzimanoglou, A., Berg, A. T., Brodie, M. J., Allen Hauser, W., Mathern, G., et al. (2010). Definition of drug resistant epilepsy: consensus proposal by the ad hoc Task Force of the ILAE Commission on Therapeutic Strategies. Epilepsia 51, 1069-1077. doi: 10.1111/j.1528-1167.2009.02397.x

Lachaux, J. P., Rudrauf, D., and Kahane, P. (2003). Intracranial EEG and human brain mapping. J. Physiol. Paris 97, 613-628. doi: 10.1016/j.jphysparis.2004.01.018

LaViolette, P. S., Rand, S. D., Ellingson, B. M., Raghavan, M., Lew, S. M., Schmainda, K. M., et al. (2011a). 3D visualization of subdural electrode shift as measured at craniotomy reopening. Epilepsy Res. 94, 102-109. doi: 10.1016/j.eplepsyres.2011.01.011

LaViolette, P. S., Rand, S. D., Raghavan, M., Ellingson, B. M., Schmainda, K. M., and Mueller, W. (2011b). Three-dimensional visualization of subdural electrodes for presurgical planning. Neurosurgery 68, 152-161. doi: 10.1227/neu.0b013e31820783ba

Lee, J. Y., Kim, J. W., Lee, J. Y., Lim, Y. H., Kim, C., Kim, D. G., et al. (2010). Is MRI a reliable tool to locate the electrode after deep brain stimulation surgery? Comparison study of CT and MRI for the localization of electrodes after DBS. Acta Neurochir. (Wien). 152, 2029-2036. doi: 10.1007/s00701-010-0779-2

Litvak, V., Mattout, J., Kiebel, S., Phillips, C., Henson, R., Kilner, J., et al. (2011). EEG and MEG data analysis in SPM8. Comput. Intell. Neurosci. 2011:852961. doi: 10.1155/2011/852961

Maes, F., Collignon, A., Vandermeulen, D., Marchal, G., and Suetens, P. (1997). Multimodality image registration by maximization of mutual information. IEEE Trans. Med. Imaging 16, 187-198. doi: 10.1109/42.563664

Maris, E., and Oostenveld, R. (2007). Nonparametric statistical testing of EEG- and MEG-data. J. Neurosci. Methods 164, 177-190. doi: 10.1016/j.jneumeth.2007.03.024

McGonigal, A., Bartolomei, F., Régis, J., Guye, M., Gavaret, M., TrébuchonDa Fonseca, A., et al. (2007). Stereoelectroencephalography in presurgical assessment of MRI-negative epilepsy. Brain 130, 3169-3183. doi: 10.1093/brain/awm218

Moshé, S. L., Perucca, E., Ryvlin, P., and Tomson, T. (2015). Epilepsy: new advances. Lancet 385, 884-898. doi: 10.1016/S0140-6736(14)60456-6

Mukamel, R., and Fried, I. (2012). Human intracranial recordings and cognitive neuroscience. Annu. Rev. Psychol. 63, 511-537. doi: 10.1146/annurev-psych-120709-145401

Nichols, T. E., and Holmes, A. P. (2001). Nonparametric permutation tests for functional neuroimaging: a primer with examples. Hum. Brain Mapp. 15, 1-25. doi: 10.1002/hbm.1058

Oostenveld, R., Fries, P., Maris, E., and Schoffelen, J.-M. (2011). FieldTrip: open source software for advanced analysis of MEG, EEG, and invasive electrophysiological data. Comput. Intell. Neurosci. 2011:156869. doi: $10.1155 / 2011 / 156869$

Phillips, H. N., Blenkmann, A., Hughes, L. E., Kochen, S., Bekinschtein, T. A., CamCAN, et al. (2016). Convergent evidence for hierarchical prediction networks from human electrocorticography and magnetoencephalography. Cortex 82, 192-205. doi: 10.1016/j.cortex.2016.05.001

Pieters, T. A., Conner, C. R., and Tandon, N. (2013). Recursive grid partitioning on a cortical surface model: an optimized technique for the localization of implanted subdural electrodes. J. Neurosurg. 118, 1086-1097. doi: 10.3171/2013.2.JNS121450

Pluim, J. P., Maintz, J. B., and Viergever, M. A. (2003). Mutual-informationbased registration of medical images: a survey. IEEE Trans. Med. Imaging 22, 986-1004. doi: 10.1109/TMI.2003.815867

Princich, J. P., Wassermann, D., Latini, F., Oddo, S., Blenkmann, A. O., Seifer, G., et al. (2013). Rapid and efficient localization of depth electrodes and cortical labeling using free and open source medical software in epilepsy surgery candidates. Front. Neurosci. 7:260. doi: 10.3389/fnins.2013.00260

Roberts, D. W., Hartov, A., Kennedy, F. E., Miga, M. I., and Paulsen, K. D. (1998). Intraoperative brain shift and deformation: a quantitative analysis of cortical displacement in 28 cases. Neurosurgery 43, 749-758. doi: 10.1097/00006123-199810000-00010

Rosenow, F., and Lüders, H. (2001). Presurgical evaluation of epilepsy. Brain 124, 1683-1700. doi: 10.1093/brain/124.9.1683

Sebastiano, F., Di Gennaro, G., Esposito, V., Picardi, A., Morace, R., Sparano, A., et al. (2006). A rapid and reliable procedure to localize subdural electrodes in presurgical evaluation of patients with drug-resistant focal epilepsy. Clin. Neurophysiol. 117, 341-347. doi: 10.1016/j.clinph.2005. 10.005

Smith, S. M. (2002). Fast robust automated brain extraction. Hum. Brain Mapp. 17, 143-155. doi: 10.1002/hbm.10062

Studholme, C., Hawkes, D. J., and Hill, D. L. (1998). "Normalized entropy measure for multimodality image alignment," in Proceedings of SPIE, ed K. M. Hanson (San Diego, CA), 132-143. doi: 10.1117/12.310835

Studholme, C., Novotny, E., Zubal, I. G., and Duncan, J. S. (2001). Estimating tissue deformation between functional images induced by intracranial electrode implantation using anatomical MRI. Neuroimage 13, 561-576. doi: 10.1006/nimg.2000.0692

Taimouri, V., Akhondi-Asl, A., Tomas-Fernandez, X., Peters, J. M., Prabhu, S. P., Poduri, A., et al. (2014). Electrode localization for planning surgical resection of the epileptogenic zone in pediatric epilepsy. Int. J. Comput. Assist. Radiol. Surg. 9, 91-105. doi: 10.1007/s11548-013-0915-6

Tao, J. X., Hawes-Ebersole, S., Baldwin, M., Shah, S., Erickson, R. K., and Ebersole, J. S. (2009). The accuracy and reliability of 3D CT/MRI coregistration in planning epilepsy surgery. Clin. Neurophysiol. 120, 748-753. doi: 10.1016/j.clinph.2009.02.002

Vadera, S., Mullin, J., Bulacio, J., Najm, I., Bingaman, W., and GonzalezMartinez, J. (2013). Stereoelectroencephalography following subdural grid placement for difficult to localize epilepsy. Neurosurgery 72, 723-729. doi: 10.1227/NEU.0b013e318285b4ae

Van Rooijen, B. D., Backes, W. H., Schijns, O. E. M. G., Colon, A., and Hofman, P. A. M. (2013). Brain imaging in chronic epilepsy patients after depth electrode (stereoelectroencephalography) implantation: magnetic resonance imaging or computed tomography? Neurosurgery 73, 543-549. doi: 10.1227/01.neu.0000431478.79536.68

Wellmer, J., Von Oertzen, J., Schaller, C., Urbach, H., König, R., Widman, G., et al. (2002). Digital photography and 3D MRI-based multimodal imaging for individualized planning of resective neocortical epilepsy surgery. Epilepsia 43, 1543-1550. doi: 10.1046/j.1528-1157.2002. 30002.x

Winkler, P. A., Vollmar, C., Krishnan, K. G., Pfluger, T., Brückmann, H., and Noachtar, S. (2000). Usefulness of 3-D reconstructed images of the human cerebral cortex for localization of subdural electrodes in epilepsy surgery. Epilepsy Res. 41, 169-178. doi: 10.1016/S0920-1211(00)0 0137-6

Yang, A. I., Wang, X., Doyle, W. K., Halgren, E., Carlson, C., Belcher, T. L., et al. (2012). Localization of dense intracranial electrode arrays using magnetic resonance imaging. Neuroimage 63, 157-165. doi: 10.1016/j.neuroimage.2012.06.039

Conflict of Interest Statement: The authors declare that the research was conducted in the absence of any commercial or financial relationships that could be construed as a potential conflict of interest.

Copyright (® 2017 Blenkmann, Phillips, Princich, Rowe, Bekinschtein, Muravchik and Kochen. This is an open-access article distributed under the terms of the Creative Commons Attribution License (CC BY). The use, distribution or reproduction in other forums is permitted, provided the original author(s) or licensor are credited and that the original publication in this journal is cited, in accordance with accepted academic practice. No use, distribution or reproduction is permitted which does not comply with these terms. 